\title{
Representation of Waveform Periodicity in the Auditory Midbrain of the Bullfrog, Rana catesbeiana
}

\author{
Andrea Megela Simmons, Mark I. Sanderson, and Catherine E. Garabedian \\ Departments of Psychology and Neuroscience, Brown University, Providence, Rhode Island 02912
}

Received: 23 November 1999; Accepted: 19 January 2000; Online publication: 31 May 2000

\begin{abstract}
The period of complex signals is encoded in the bullfrog's eighth nerve by a synchrony code based on phase-locked responding. We examined how these arrays of phase-locked activity are represented in different subnuclei of the auditory midbrain, the torus semicircularis (TS). Recording sites in different areas of the TS differ in their ability to synchronize to the envelope of complex stimuli, and these differences in synchronous activity are related to response latency. Cells in the caudal principal nucleus (cell sparse zone) have longer latencies, and show little or no phaselocked activity, even in response to low modulation rates, while some cells in lateral areas of the TS (magnocellular nucleus, lateral part of principal nucleus) synchronize to rates as high as $90-100 \mathrm{~Hz}$. At midlevels of the TS, there is a lateral-to-medial gradient of synchronization ability: cells located more laterally show better phaselocking than those located more medially. Pooled all-order interval histograms from short latency cells located in the lateral TS represent the waveform periodicity of a biologically relevant complex harmonic signal at different stimulus levels, and in a manner consistent with behavioral data from vocalizing male frogs. Long latency cells in the caudal parts of the TS (cell sparse zone, caudal magnocellular nucleus) code stimulus period by changes in spike rate, rather than by changes in synchronized activity. These data suggest that neural codes based on rate processing and time domain processing are represented in anatomically different areas of the TS. They
\end{abstract}

Correspondence to: Dr. Andrea M. Simmons - Brown University • Department of Psychology • Box 1853 - Providence, RI 02912. Telephone: (401) 863-2283; fax: (401) 863-1074; e-mail: Andrea_Simmons@brown.edu further show that a populationbased analysis can increase the precision with which temporal features are represented in the central auditory system.

Keywords: auditory, midbrain, temporal coding, periodicity coding, synchrony, complex sounds

\section{INTRODUCTION}

Waveform periodicity or "pitch" is a prominent perceptual cue for recognition and discrimination of complex sounds, including speech, music, and animal communication sounds. Pitch may be a "primitive" auditory attribute, in the sense that it is shared by many species. Behavioral studies show that even fishes and frogs perceive acoustic features that are important in conveying a sense of pitch to humans (reviewed in Fay and Simmons 1999). For example, some species of frogs can detect changes in the periodicity of complex signals produced by changes in amplitude modulation (AM) rate, phase spectrum, or mistuning of individual harmonics (Gerhardt 1978; Allan and Simmons 1994; Bodnar 1996; Hainfeld et al. 1996; Simmons and Bean 2000; reviewed in Fay and Simmons 1999). Male and female green tree frogs (Hyla cinerea) use the cue of waveform periodicity to distinguish between their species' advertisement and aggressive calls (Gerhardt 1978; Allan and Simmons 1994); moreover, discrimination of period is equally precise for AM noise and AM tones, within the biologicallyrelevant modulation range for this species $(50-300 \mathrm{~Hz}$; Allan and Simmons 1994). Male bullfrogs (Rana catesbeiana) are sensitive to changes in the periodicity and in the fine-structure of their species advertisement calls, when these finestructure changes produce corresponding fluctuations 
in the overall stimulus envelope (Hainfeld et al. 1996). The ability of males to detect fluctuations in the stimulus envelope is limited to modulation rates lower than $200 \mathrm{~Hz}$, but appears to be invariant with stimulus level (Simmons and Bean 2000).

Physiological studies in different vertebrate species demonstrate that the waveform periodicity of complex acoustic signals, over a wide frequency range, is encoded in the cycle-by-cycle phase-locked activity of eighth nerve fibers (reviews: Langner 1992, Simmons and Buxbaum 1996). In particular, the periodicity and fundamental frequency of AM signals and multipleharmonic complex sounds are represented in the synchronized discharge patterns of eighth nerve fibers in mammals (e.g., Evans 1983; Cariani and Delgutte 1996a,b), fish (Fay 1980) and frogs (Schwartz and Simmons 1990; Simmons et al. 1993). Moreover, both in cats and in frogs, temporal discharge patterns of eighth nerve fibers, measured either as period histograms or all-order interval histograms, reflect changes in the perceived pitch of complex signals, such as occur under conditions of pitch shift or changes in component phase (Simmons and Ferragamo 1993; Simmons et al. 1993; Cariani and Delgutte 1996b). In the bullfrog's auditory nerve, the representation of waveform periodicity by all-order interval histograms is robust under conditions of background noise, where the rate response is saturated (Simmons et al. 1992). These data are consistent with the hypothesis that pitch corresponds to the most frequent time interval present in synchronized responses of eighth nerve fibers (Licklider 1951; Meddis and Hewitt 1991; Cariani 1999).

In contrast to the large body of research on coding of periodicity in the peripheral auditory system, relatively less is known about the representation of pitch in central auditory nuclei (reviews: Langner 1992; Lyon and Shamma 1996). Neurophysiological data on central representation of periodicity are important for devising biologically realistic computational models of pitch that are not restricted to events in the eighth nerve, and for testing various models of pitch formation based primarily on human psychoacoustic data (Lyon and Shamma 1996; Cariani 1999). Phase-locked activity in the cochlear nucleus can be robust (Langner 1992; Rhode and Greenberg 1994; Rhode 1995), and interspike interval distributions can represent the periodicity of complex stimuli in some types of neurons, in a manner that reflects psychoacoustic phenomena such as pitch shift (Kim et al. 1990; Rhode 1995). The main obstacle for a temporally-based model of pitch in the central auditory system, particularly above the level of the medulla, is the common observation that the range of phase-locked activity is generally more restricted in central nuclei than in the eighth nerve (Langner 1992). The degradation of synchronized activity becomes pronounced at the level of the inferior colliculus (IC), where only rare neurons exhibit synchronized activity to AM rates above about $200-300$ $\mathrm{Hz}$ (Rees and Moller 1987; Langner and Schreiner 1988; Langner 1992). The restricted range of phaselocked activity in the IC led to the view that a central synchrony code cannot be useful in identifying or classifying complex sounds (Eggermont 1990) and to proposals that a neural code based on phase-locking is transformed in this nucleus into a code based on spike rate responses. Rose and Capranica $(1984,1985)$ first showed that the periodicity of AM signals is encoded in the frog's auditory midbrain (torus semicircularis, TS), not by changes in synchronized neural response with changes in AM rate, but by changes in the shapes and best sensitivities of spike rate tuning curves compiled over AM rate. They identified 5 different classes of rate-based AM tuning curves, one class of which shows band pass tuning to particular AM rates. These band pass neurons were viewed as "matched temporal filters" for processing the periodicity of the frog's communication sounds (Rose and Capranica 1984). On a broader level, these band pass (AM tuned) neurons might function as an array of filters tuned to specific pitches (modulation rates) in complex signals (Langner 1992). The existence of band pass neurons in the auditory midbrain has been confirmed in frogs (e.g., Epping and Eggermont 1986a,b; Eggermont 1990; Gooler and Feng 1992; Diekamp and Gerhardt 1995) and also identified in mammals (e.g., Rees and Moller 1987; Langner and Schreiner 1988), although different studies provide different estimates on the relative number of these types of neurons. Schreiner and Langner (1988) further demonstrated an explicit mapping of neurons with different best modulation frequencies along isofrequency planes in the IC, reflecting a "periodotopic" mapping orthogonal to tonotopic mapping. Such an explicit spatial representation of AM rate has not been demonstrated in frogs. Cariani (1999) and Cariani and Delgutte (1996b) have pointed out some problems with a processing scheme for pitch based solely on band pass neurons. In particular, tuning of these neurons is influenced by stimulus properties such as level, rise/fall time, duration, and the presence of background noise (Rees and Moller 1987; Rees and Palmer 1989; Gooler and Feng 1992; Diekamp and Gerhardt 1995; Simmons and Buxbaum 1996). Thus, whether response properties of AM-tuned neurons can fully account for behavioral performance in pitch tasks, by either humans or animals, is not clear.

Alternative metrics for processing of pitch in the central auditory system that may work in parallel with or in addition to such an array of band pass filters have also been proposed. Langner (1983, 1992; Langner and Schreiner 1988) identified intrinsic oscillations in the responses of IC cells in both the guinea 
fowl and the cat, and incorporated these response properties into a coincidence model of pitch formation in the IC which includes band pass neurons. Cariani (1999) proposed a model of pitch extraction based on population interval coding in the central auditory system. This model hypothesizes that a populationbased analysis that measures time intervals between responses of groups of neurons can be used to extract the periodicity of complex sounds with high precision, bypassing the limit in phaselocking ability of individual cells. There are few physiological data explicitly in support of either model.

Here, we report experiments designed to examine the representation of waveform periodicity in the bullfrog's TS, using metrics based on rate coding, synchronous activity of individual neurons, and population interval coding. The bullfrog is an excellent model for analysis of neural mechanisms of complex auditory phenomena, because of the ability to collect both physiological and behavioral data on the same species (Simmons and Buxbaum 1996). The periodicity of complex acoustic signals is extracted in the bullfrog's auditory periphery by a temporal code based on phase-locked discharges (Simmons and Buxbaum 1996), and rate tuning in the TS has been well-described (Rose and Capranica 1984, 1985; Epping and Eggermont 1986a,b; Eggermont 1990; Gooler and Feng 1992; reviewed in Feng and Schellart 1999). Although a few studies have mentioned the existence of highly phaselocked cells in the TS (Epping and Eggermont 1986a,b; Rose and Capranica 1985), most earlier work has focused primarily on the responses of band pass neurons and has not provided a quantitative analysis of temporal response properties. More recently, Bibikov and Nizamov (1996) showed that TS cells strongly phase lock to AM rates of $20 \mathrm{~Hz}$, even at low depths of modulation. Although they called for a re-evaluation of the predominant view that TS cells show weak phaselocking, they did not test higher modulation rates within the frog's range of behavioral sensitivity to AM.

We examined the representation of waveform periodicity in different regions of the bullfrog's TS, using metrics based on both rate and interval coding, on both single unit and population levels. The anatomical distribution of AM-tuned neurons in the frog's TS has not been described, and time interval coding by TS cells has not been systematically examined on either physiological or anatomical levels (reviews: Simmons and Buxbaum 1996, Feng and Schellart 1999). Our results indicate that some neurons in subnuclei of the TS show a greater range of synchronized activity to certain kinds of complex signals than has been previously reported for anurans. Our data also show that the period of complex signals can be represented by groups of cells in the lateral part of the TS by a time interval code, while cells in the more caudal and medial parts of the TS code stimulus period by a rate code. This suggests that different neural codes for periodicity are expressed by neurons in anatomically distinct areas of the TS.

\section{MATERIALS AND METHODS}

\section{Recording procedures}

Experimental protocols were approved by the Brown University Institutional Animal Use and Care Committee. Adult bullfrogs (snout-vent length $>10 \mathrm{~cm}$, weight $150-400 \mathrm{~g}$ ) were obtained from a commercial supplier (Charles Sullivan, Nashville, TN). Animals were anesthetized for surgery by immersion in a bath of $0.1 \%$ 3-aminobenzoic acid ethyl ester (MS-222, Sigma, St. Louis, MO). We exposed the surface of the optic tectum by drilling through overlying skull and retracting the dura and pia membranes. The wound was packed with antiseptic gelfoam, and its margins were swabbed with topical anesthetic (Lidocaine). To avoid possible confounds in the data due to differences between animals in depth of or rapidity of recovery from anesthesia, animals were allowed to recover for 24 hours prior to electrophysiological recordings.

On the day of experimentation, the animal was immobilized by injection of d-tubocurarine chloride $(0.006 \mathrm{mg} / \mathrm{g}$; Sigma), and wrapped in wet gauze to facilitate cutaneous respiration. The margins of the wound were swabbed with topical anesthetic, the gelfoam was removed, and the optic tectum was exposed. The animal was placed on a styrofoam platform located on a vibration isolation table in a sound attenuating chamber. The frog's body temperature was maintained within the range of $20-22^{\circ} \mathrm{C}$. Neural activity was recorded with tungsten microelectrodes (9-11 MW; FHC, Bowdoinville, ME) or glass micropipettes filled with $10-30 \%$ unconjugated horseradish peroxidase (HRP; Sigma). The electrodes were positioned on the surface of the optic tectum, and advanced through the tectum using an oil-driven microdrive (Trent-Wells, Coulterville, CA). Negative holding current $(0.5 \mu \mathrm{A})$ was applied to HRP electrodes to prevent leakage. Neural activity was differentially amplified (WPI; $0.3-3$ $\mathrm{kHz}$, gain of 10,000), bandpass filtered from 300-3000 $\mathrm{Hz}$ and amplified (Rockland; $20 \mathrm{~dB}$ gain), and displayed on an oscilloscope. Action potentials were passed through a custom-built window discriminator and Schmitt trigger with a signal-to-noise ratio set at a criterion level of 4:1 to capture spike times. The output of the window discriminator was digitally converted (R/C Electronics, Santa Barbara, CA) at a sampling interval of $100 \mu \mathrm{s}$ (sampling rate of $10 \mathrm{kHz}$ ), displayed as dot rasters, and saved to hard disk. Both single and multiple unit activity were recorded with 
both types of electrodes. When multiple unit activity was encountered, the level of the Schmitt trigger was set high enough to capture only the peaks of the largest amplitude spikes, representing activity from an estimated 2-4 units. Neural activity and stimuli were also recorded on analog tape for additional off-line analyses. Recording sessions lasted from 3 to 6 hours.

\section{Stimulus generation and delivery}

Acoustic stimuli were digitally generated using customwritten software on a Pentium computer using an arbitrary function generator and digital-to-analog converter (R/C Electronics 200 series) with 16-bit resolution. Sampling rate for digital generation was $16 \mathrm{kHz}$. Signals were stored on computer for subsequent recall during an experiment. Two different stimulus sets, AM noise and pulsed noise, were used; these stimuli are useful for studies of time-domain coding because they contain no spectral cues. AM noise series consisted of bursts of frozen broadband noise (frequency range $100-7000 \mathrm{~Hz}$ ) modulated with a cosine function at rates from 10 to $200 \mathrm{~Hz}$. Frozen noise was used to ensure an identical carrier stimulus at any given AM rate; the long-term spectrum of this stimulus was flat. The range of AM rates chosen corresponds with the range of rates used in previous studies in the frog's auditory midbrain (reviewed in Feng and Schellart 1999) and spans the biologicallyrelevant AM rates for the bullfrog (Hainfeld et al. 1996). Preliminary data showed that synchronization was not statistically significant at rates beyond $100 \mathrm{~Hz}$ at any recording site, so in most experiments the range of $\mathrm{AM}$ rates presented was limited to $10-100 \mathrm{~Hz}$. The use of a cosine gating function resulted in the initial modulating cycle for each AM rate beginning at the peak in the cycle (see Fig. 2); this eliminated differences in onset latency to different AM rates resulting from differences in risetime. Modulation depth was maintained at $100 \%$. Stimulus duration was $200 \mathrm{~ms}$ and rise/fall time was $2 \mathrm{~ms}$. Pulsed noise consisted of 10-ms noise bursts pulsed at rates from 10 to $90 \mathrm{~Hz}$ in a 300-ms window. Rise/fall time of each pulse was $1 \mathrm{~ms}$. AM noise and pulsed noise bursts convey a percept of periodicity pitch for humans (Flanagan and Gutmann 1960; Burns and Viemeister 1976). Frogs can also discriminate AM noise bursts based on modulation rate (Allan and Simmons 1994).

A complex stimulus without a noise-like time waveform was also presented in a subset of experiments, in order to estimate any differences between the extent of synchronized responses to noise-like and spectrallystructured stimuli. The complex stimulus used was a synthetic version of a male bullfrog's advertisement call. Behavioral experiments (Hainfeld et al. 1996; Simmons and Bean 2000) show that the periodicity of this signal is crucial for mediating the evoked vocal response of male bullfrogs. The stimulus consisted of 21 frequency components, ranging from 100 to 2200 $\mathrm{Hz}$, at $100-\mathrm{Hz}$ intervals, added together in sine phase; its amplitude spectrum and autocorrelation function (ACF) are shown in Figure 8. Stimulus duration was $800 \mathrm{~ms}$ and rise/fall time was $50 \mathrm{~ms}$. This stimulus is similar to that used in both physiological (Schwartz and Simmons 1990; Simmons et al. 1993) and behavioral (Hainfeld et al 1996; Simmons and Bean 2000) studies of periodicity perception in bullfrogs.

Stimuli were low-pass filtered (Krohn-Hite 3550) at $7 \mathrm{kHz}$, attenuated (Coulbourn S85-05 electronic attenuator and Leader manual attenuator), passed through a stereo equalizer (Rane), and amplifier (Harman/Kardon PM 645). They were presented to the bullfrog's external tympanum contralateral to the recording site using a Beyer DT 48 earphone enclosed in a custom-built brass housing. A tapered rubber tube attached to the face of this housing was placed close to, but not touching, the tympanum, and sealed around its edges with silicone grease. The earphone housing also contained a 1-mm-diameter probe tube attached to a 1/2-in. condenser microphone (model 4134, Bruel and Kjaer, Norcross GA) for monitoring stimulus amplitudes. The condenser microphone was attached to a Bruel and Kjaer 2209 sound level meter located outside the recording chamber. The frequency response of the sound delivery system was calibrated before each experimental session using a $\mathrm{R} / \mathrm{C}$ Electronics isc- 16 interface board and custom-written software. The system had a flat $( \pm 3 \mathrm{~dB})$ frequency response over the range of $100-5000 \mathrm{~Hz}$. This closedfield system was used to facilitate comparisons with peripheral fiber work on coding of stimulus periodicity in the bullfrog (Schwartz and Simmons 1990; Simmons et al. 1993), and with previous studies of AM coding in the frog's auditory midbrain (Rose and Capranica 1985; Gooler and Feng 1992).

\section{Experimental protocol}

During an experiment, an unmodulated frozen noise burst (200 ms duration, $80 \mathrm{~dB}$ SPL re: $20 \mu \mathrm{Pa}$ ) was used as a search stimulus. When an acoustically responsive site was encountered, its threshold to the search stimulus was estimated by a justnoticeable increase in firing rate monitored on an oscilloscope and audio monitor. Responses to the search stimulus at $20 \mathrm{~dB}$ above estimated threshold were then recorded. Best frequency to tone bursts was estimated only for a small subset of cells. AM or pulsed noise bursts were presented as a set, within which the different rates were presented in random order. The order of presentation of AM or pulsed noise was counterbalanced across 
experiments. All stimuli were presented for 16 repetitions (interstimulus interval $2800 \mathrm{~ms}$ ) at $20 \mathrm{~dB}$ above threshold. If time permitted, the experiment was repeated for stimulus intensities of $10 \mathrm{~dB}$ and $30 \mathrm{~dB}$ above threshold. At the end of stimulus presentation, recording sites were marked with an electrolytic lesion (2.5 $\mu \mathrm{A}$ negative current for $5 \mathrm{~min}$ ) or deposition of HRP (positive current of $1.5 \mu \mathrm{A}$ for 5-10 min). Only data from marked and recovered recording sites are included in this report.

We collected data from and marked 1-3 recording sites per frog brain. In experiments using HRP-filled electrodes, only one site was marked in order to unambiguously trace HRP transport patterns. In experiments using tungsten electrodes, we chose to mark a small number (typically 1-3) of recording sites per brain. We noted that our ability to identify clear single unit activity was inversely related to the depth of the animal's paralysis: Animals that were only lightly immobilized yielded clearer single unit activity. The downside of this was that the animals tended to exhibit reflex activity and slight movements that impeded long-term stable recordings. Supplemental dosages of curare often led to deep paralysis and decreased the prevalence of clear single unit activity. We therefore deliberately opted for shorter recording sessions with lightly immobilized animals and a limited number of recording sites per brain.

\section{Data analysis}

Custom-written software was used to analyze spike train responses. Peristimulus time (PST) histograms (1 ms bin width) were compiled to estimate spike count (total spikes per stimulus). Response latency was quantified in two ways. First, latency to the first spike was calculated for each stimulus repetition, and these "raw" latencies were then averaged over the 16 repetitions of each stimulus. The use of the average latency over 16 repetitions eliminates the influence of spontaneous spikes occurring earlier in time than evoked spikes, although high levels of spontaneous activity might still result in inaccurate estimates of latency. Second, we used an algorithm adapted from Ferragamo et al. (1998) that calculated the latency to the initial peak in the PST histogram based on cumulative distributions of spike times; latency is defined as the first bin in which the cumulative PST crosses a fixed threshold level. This measure of latency is less likely to be influenced by spontaneous spikes. Both latency measures were calculated using a $100-\mu$ s bin width. A cell's response latency to a given stimulus type at a fixed amplitude was taken from the median of all available first spike latencies to different modulation rates.

Rate modulation transfer functions (rMTF) were calculated by plotting normalized spike count (total spikes/stimulus, normalized to the highest number of spikes for a particular stimulus type) as a function of AM rate or pulse rate. The shapes of rMTFs were classified following the criteria in Rose and Capranica (1985). In particular, a band pass rMTF is defined as one in which a maximum number of spikes occurs to a particular (preferred) AM rate, while the number of spikes to both lower and higher (nonpreferred) $\mathrm{AM}$ rates is at most $50 \%$ of that to the preferred rate. Band-suppression rMTFs show maximal spike counts to low and high AM rates, but weak responses $(<50 \%$ of the maximum) to intermediate rates. Low-pass and high-pass rMTFs have maximal spike counts to low and high modulating rates, respectively, with spike counts dropping to at least $50 \%$ of the maximum at other rates. If spike count is independent of AM; rate, then the rMTF is described as nonselective. Temporal discharge patterns were analyzed by compiling period histograms (200 $\mu$ s bin width), using AM or pulse rate as the period length. Vector strength (VS; equivalent to the synchronization index) was calculated from the period histogram to quantify degree of response synchrony to the period of the stimulus. VS ranges from 0 , indicating no synchrony, to 1 , indicating perfect phase locking (all responses in one bin). The Rayleigh test of uniformity (Batschelet 1981) was used to determine the statistical significance [likelihood value $\left(n \mathrm{VS}^{2}\right)>13.8, p<0.001$, where $n=$ number of spikes[ of each VS value. Because number of spikes is used to calculate statistical significance, high values of VS obtained from cells with low spike counts tend to be nonsignificant; low Rayleigh values would also be obtained from recordings in which spike times are highly variable and/or not related to the stimulus modulation rate. Synchronization MTFs (sMTF) were compiled by plotting VS against AM rate or pulse rate. The highest AM rate or pulse rate at which VS was statistically significant was also quantified for each recording site.

All-order interspike interval histograms (the autocorrelation function, ACF) were generated using MATLAB v. 5.2 (MathWorks Inc., Natick, MA) scripts. These histograms are calculated by measuring the time intervals between both successive and nonsuccessive spikes in the neural response, over the entire duration of the stimulus (Moller 1970), for both single unit and multiunit responses. ACFs are useful tools for studying temporal coding, because, unlike period histograms, they do not require prior knowledge of the period of the stimulus. ACFs were calculated using bin widths of 100 $\mu$ s. ACFs at individual recording sites were added up to yield a pooled population interval histogram (Cariani and Delgutte 1996a). Individual ACFs were not weighted in any way for pooling. Peaks in the pooled ACF were identified visually and compared to the ACF of the stimulus. If pitch is extracted by time domain 
processing, then the peak in the pooled ACF, corresponding to the most predominant interval in the interval distribution, would be expected to occur at the stimulus period (Meddis and Hewitt 1991).

Statistical tests were performed using SPSS for Windows, Rel. 7.5.1 (SPSS Inc., Chicago, IL).

\section{Histology}

At the end of a recording session, animals in which neural activity was recorded with tungsten electrodes were terminally anesthetized by immersion in MS-222. They were transcardially perfused with heparinated $0.9 \%$ saline followed by a $4 \%$ solution of glutaraldehyde in $0.1 \mathrm{M}$ phosphate buffer ( $\mathrm{pH} 7.45$ ). The brains were removed and stored in $4 \%$ glutaraldehyde in buffer. Tissue was embedded in a mixture of albumin and gelatin polymerized with $25 \%$ glutaraldehyde, sliced at $50 \mu \mathrm{m}$ thickness, and stained with cresyl violet. Animals in which HRP deposits were made were allowed to recover for 2-4 days after recording, and were then terminally anesthetized and perfused as described above. HRP was visualized using a modified Hanker-Yates method (Hanker et al. 1977). Alternate sections were counterstained with cresyl violet to allow localization of injection sites.

Electrolytic lesions were identified using an Olympus $\mathrm{BH} 2$ microscope $(40 \times)$. Brain sections containing a lesion site were stored on a computerized imaging system. Brain sections were digitally imaged through an Olympus BH2 microscope connected to a $166 \mathrm{MHz}$ Pentium computer running Windows 95, via a Hitachi KP-D50 Digital Video camera and a Flashpoint image acquisition board. Sections were acquired, spatially and density calibrated using Scion Image and ImagePro 3.0 analysis software, stored as TIFF files, and postprocessed for contrast enhancement and removal of embedding artifacts. Section outlines were generated from scanned images by outline tracing followed by low-pass filtering. We made composite figures overlying locations of recording sites from all brains by using two major criteria. In the rostral/caudal dimension, we used distance from the last appearance of the nucleus isthmus as a marker. In the medial/lateral dimension, we used relative distance from the midpoint of the TS, where the two lobes join. Composite drawings were made independently of physiological characterization of a particular site.

\section{Anatomical nomenclature}

The TS consists of 5 different subnuclei, 3 of which respond to sounds. These 3 nuclei (the principal nucleus, $\mathrm{Tp}$; the laminar nucleus, $\mathrm{Tl}$, and the magnocellular nucleus, Tmc) differ in patterns of afferent and efferent input, and in cell morphology (Feng 1983; Wilczynski 1988; Feng and Lin 1991; Luksch and Walkowiak 1998). In spite of these anatomical differences, most physiological studies, with the exception of those related specifically to tonotopy (reviewed in Feng and Schellart 1999), have not featured histological verification of all recording sites. Thus, systematic relationships between response properties and spatial location within subnuclei of the TS cannot be derived from these previous data. Rose and Capranica (1985) reported that band pass neurons were located primarily in the caudal area of the TS termed the cell sparse zone (csz, see below), but they did not provide quantitative data on response properties in other areas of the TS. Alder and Rose (1998) reported that band pass neurons with long latencies were found in the medial TS, but they provided no histological verification of recording sites.

Locations of recording sites in this report are classified with reference to the terminology and approximate boundaries described in Wilczynski 1988 and Feng and Lin (1991); see Fig. 1. The Tp, Tl, and Tmc vary in dimensions and in boundaries over the caudalto-rostral extent of the midbrain. We define caudal TS as beginning at the level of the nucleus isthmus and the interpeduncular nucleus, and rostral TS as the area at the level of the oculomotor nucleus, at the merging of the optic ventricle and the aqueduct of Sylvius. Midway between these two extremes, we refer to midlevels of the TS. Tl consists of the alternating cell and fiber layers directly below the optic ventricle and extends throughout caudal up to rostral TS. Tp is located ventral to $\mathrm{Tl}$ and dorsal to the nucleus isthmus at caudal levels, and to the tegmentum at medial and rostral levels. This area becomes more cell dense from caudal up to rostral areas. At caudal levels, up to approximately $200 \mu \mathrm{m}$ after the disappearance of the nucleus isthmus, the medial TS contains a few scattered cells, and thus has been termed the cell sparse zone (csz; Rose and Capranica 1985; see Fig. 1B). At midlevels, Tp is more cell dense, and these cells are typically arranged in clusters, in a pattern described as an onion skin arrangement (Feng 1983; see Fig. 1A). Tmc is located lateral to Tp and csz. The boundary between $\mathrm{Tp}$ and $\mathrm{Tmc}$ can be difficult to determine; differences in cell size, soma shape, and dendritic length are typically used to make these demarcations (Feng 1983; Luksch and Walkowiak 1998). Cells in the Tmc are large and are not densely clustered, and this region remains relatively cell sparse over the caudal-rostral extent of the TS. Wilczynski 1988 includes the caudal csz and caudal Tmc together as the ventral toral zone, while Feng and Lin (1991) include the medial cell sparse zone as part of Tp and the lateral zone as Tmc. Our data show that medial and lateral regions of the TS, even at caudal levels, 

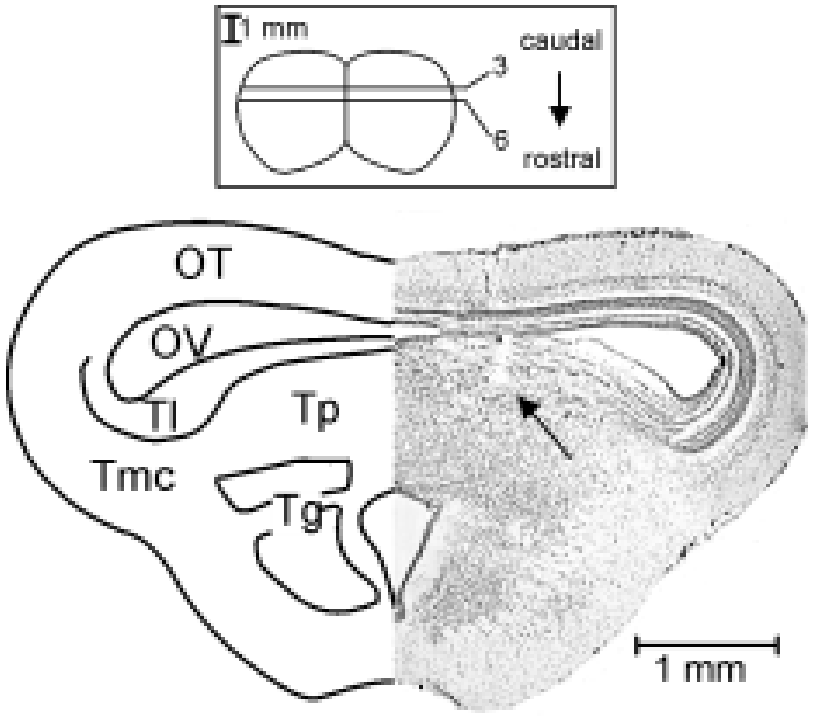

A. EL lesion: C-R level 6

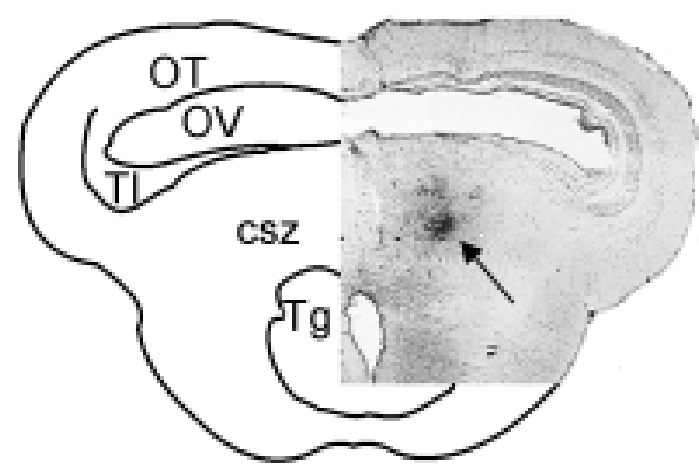

B. HRP deposit:C-R level 3

FIG. 1. Schematic scans showing (A) a typical electrolytic lesion (EL) and (B) a typical HRP deposit in the TS. On each schematic, a line drawing showing terminology used to classify recording sites is located on the left, and a digital scan of a cresyl violet-stained section is shown on the right. The schematic was drawn with CorelDraw 8 from cresyl violetstained sections (50 $\mu$ m thick) viewed through an Olympus microscope (magnification $2 \times$ ) and imaged using a computer-driven imaging system (Image Pro v. 3.0). The line drawing on the top shows a dorsal view of the frog's optic tectum, showing caudal-rostral (C-R) locations of the individual schematics. A, EL lesion, dorsal portion of the Tp (C-R level 6). B, HRP deposit in the Csz (C-R level 3). Csz = cell sparse zone; OT = optic tectum; OV $=$ optic ventricle; $\mathrm{TI}=$ laminar nucleus; $\mathrm{Tp}=$ principal nucleus; $\mathrm{Tmc}=$ magnocellular nucleus; $\mathrm{Tg}=$ tegmentum.

show different responses to complex stimuli, and that response properties in medial regions differ between caudal and midlevels of the TS. We therefore separate Tmc from csz in caudal levels, and csz from Tp along the caudal-rostral axis, using as criteria relative lateral/medial position, and differences in cell density and cell size around the lesion site.

\begin{tabular}{lccc}
\hline \multicolumn{4}{c}{ TABLE 1 } \\
\hline Number of recording sites in response to each stimulus type \\
\hline $\begin{array}{l}\text { Level above threshold } \\
\text { (dB SPL) }\end{array}$ & AM & Pulsed \\
& Noise & noise & Both \\
\hline+10 & 44 & 41 & 32 \\
+20 & 70 & 69 & 56 \\
+30 & 44 & 49 & 36 \\
\hline
\end{tabular}

\section{RESULTS}

Data were collected from a total of 104 marked and recovered recording sites (68 presumed single unit sites and 36 multiple unit sites) from the TS of 63 frogs. The number of recordings obtained for each stimulus type and level is shown in Table 1 . The relative proportion of single unit to multiple unit sites is similar at all stimulus levels, and the overall pattern of neural responding (that is, whether phaselocked or not) did not differ reliably between single and multiple unit sites within a given TS area. Figure 1 shows digital scans of typical examples of electrolytic lesions (Fig. 1A) and HRP deposits (Fig. 1B) obtained at two different rostral-caudal locations in the TS (Fig. 1, top insert).

\section{Synchronized activity}

Figure 2 shows PST histograms of responses to AM noise bursts (2A) and to pulsed noise bursts (2B) of a single unit site (middle row, $\mathrm{A}$ and $\mathrm{B}$ ) and a multiple unit site (bottom row, A and B) in the Tmc. The stimulus waveforms as delivered to the earphone are shown at the top of each column. In response to AM noise, both sites show significant phase-locking to the envelope period, up to a rate of $50 \mathrm{~Hz}$. Cycle-by-cycle phaselocked responding declined at higher rates (Fig. 2A, bottom row), although significant values of VS were sometimes obtained to AM rates as high as $90 \mathrm{~Hz}$ (middle row). Similar patterns were typically seen in response to pulsed noise (Fig. 2B), where both single unit (middle row) and multiple unit sites (bottom row) in the Tmc typically showed good phase-locked response up to rates of $50 \mathrm{~Hz}$. At higher rates, the PST histogram tends to exhibit an onset-like response, with fewer spikes in the steady-state portion of the response; the response rate is unrelated to the modulation rate, and the Rayleigh value is nonsignificant. Note that, in response to both stimulus types, high levels of synchronized activity were not merely a consequence of multiple unit recordings, but were also seen in single unit responses.

Changes in VS across AM and pulse rate at two different stimulus levels are presented in the form of 


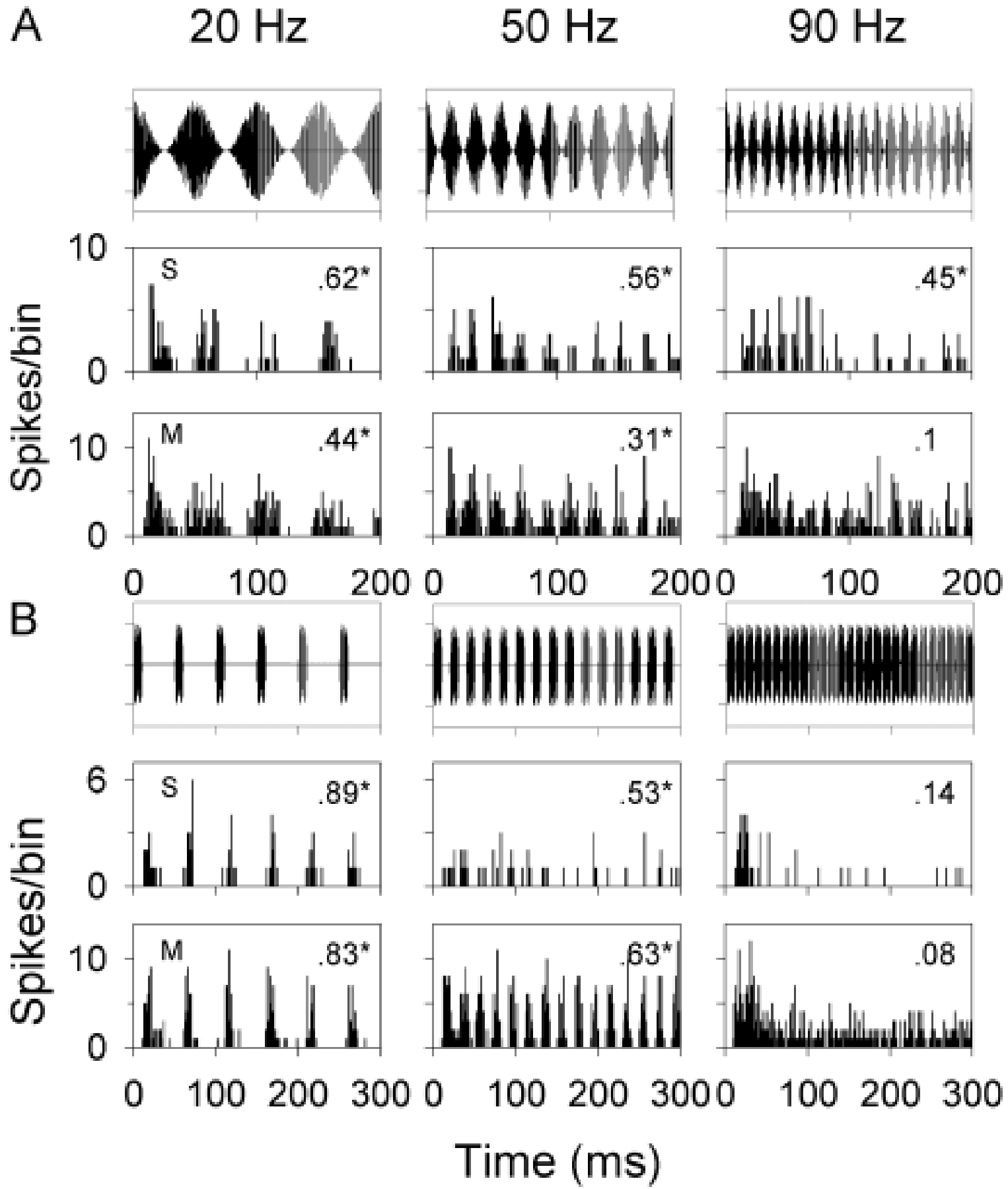

FIG. 2. PST histograms (bin width $=1 \mathrm{~ms}$ ) of responses from two different cells to AM noise (A) and to pulsed noise (B). A,B top row shows the stimulus waveforms delivered to the earphone ( $y$ axis shows relative voltage, $x$ axis shows duration). AM noise (A) has a duration of $200 \mathrm{~ms}$ and pulsed noise (B) has a duration of $300 \mathrm{~ms}$. Neural responses are shown from the same cells (middle and bottom rows).

$\mathrm{S}=$ single unit site; $\mathrm{M}=$ multiple unit site. $\mathrm{VS}$ calculated from period histograms of the response are indicated in the upper right corner of each graph. Asterisks indicate significant $(p<0.001)$ VS as indicated by the Rayleigh test. Data were collected at a stimulus level of 20 $\mathrm{dB}$ above response threshold.

sMTFs for single unit sites in the csz (Fig. 3A), Tmc (Fig. 3B), and Tp (Fig. 3C). Typically, the sMTF, regardless of recording site or stimulus type, is either low-pass or nonselective in shape. Differences in the sMTF emerge in the frequency range of significant

phase-locked activity. In the csz (Fig. 3A), some recording sites show no significant synchronous activity even at $\mathrm{AM}$ or pulse rates as low as $20 \mathrm{~Hz}$ (A1, A3); in other cases (A2, A4), synchronization is present at modulation rates of $10 \mathrm{~Hz}$, but is not statistically 


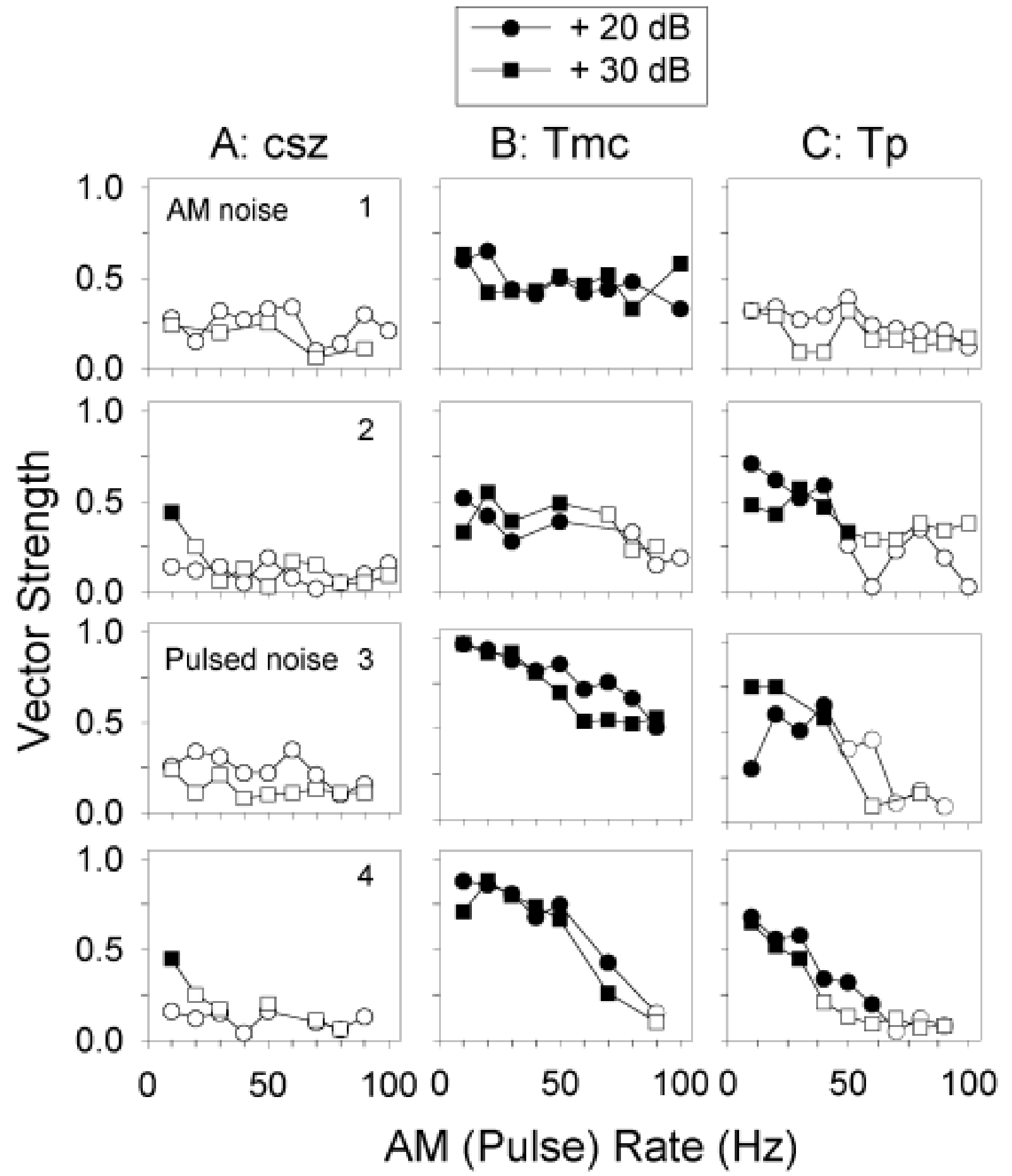

FIG. 3. Synchronization modulation transfer functions (sMTF) plotting VS against rate for AM noise (rows 1 and 2) and for pulsed noise (rows 3 and 4). Data are from 12 single unit sites in the TS, stimulated at 20 and $30 \mathrm{~dB}$ above threshold. Recording sites in column $\mathbf{A}$ are located in the csz, recording sites shown in column $\mathbf{B}$ are located in

significant at higher rates. In contrast, in the Tmc (Fig. 3B), significant synchronous activity is sometimes observed to AM or pulse rates as high as $90 \mathrm{~Hz}$ (B3) or $100 \mathrm{~Hz}$ (B1). In the Tp (Fig. 3C), synchronization appears to be intermediate between that observed in
Tmc, and recording sites in column $\mathbf{C}$ are located in Tp at midlevels. Data collected at $20 \mathrm{~dB}$ above threshold are indicated by circles and data collected at $30 \mathrm{~dB}$ above threshold are indicated by squares; see legend. Significant VS values are shown by closed symbols; nonsignificant values are shown by open symbols.

the csz and in the Tmc, with some sites showing no synchrony even to low rates of modulation (C1) and other sites showing synchrony to rates as high as 60 $\mathrm{Hz}$ (C4). The shape of the sMTF does not vary with stimulus level at any recording site (data at $+10 \mathrm{~dB}$ 
are not shown, but are consistent), and, in most cases, the frequency range of significant synchronous activity also does not vary with changes in stimulus level. sMTFs from both single unit and multiple unit recordings are similar in shape, and there were no reliable differences in the range of significant VS values found in these different types of recordings.

Regional differences in phase-locked responses were further quantified by identifying for each site the highest AM or pulse rate producing significant synchronization. Box plots of these cutoffs for synchronization, showing mean, median, and outlying values, are presented in Figure 4 for AM noise (left column) and pulsed noise (right column) at all stimulus levels tested. Although variability in cut-off frequencies were observed in different subnuclei, one way analysis of variance (ANOVA, performed on each stimulus type at each stimulus level) showed a significant main effect of site at all stimulus levels for pulsed noise, and at 20 and $30 \mathrm{~dB}$ above threshold for $\mathrm{AM}$ noise (see figure caption for $F$ values). In the case of AM noise at $10 \mathrm{~dB}$ above threshold, the ANOVA showed only a marginally significant $(p=0.1)$ effect of site. Post hoc comparisons on the significant ANOVAs were made using the Tukey HSD test. For AM noise at +20 and $+30 \mathrm{~dB}$, and for pulsed noise at all levels, the mean cutoff frequency in the csz was significantly lower $(p<0.05)$ than that in Tmc. Differences between csz and Tp sites were significant for pulsed noise at all stimulus levels and for AM noise at $+20 \mathrm{~dB}$. Differences between Tmc and $\mathrm{Tp}$ sites were significant for AM noise at +20 and +30 , but not for pulsed noise at any stimulus level. There were no significant differences in cutoffs between $\mathrm{Tp}$ and $\mathrm{Tl}$ sites at any stimulus level for either stimulus type. These data show that the most consistent differences in cutoff frequencies, regardless of stimulus type and level, are between csz and Tmc sites.

At all intensity levels tested, a large proportion of sites in the csz show no significant synchronization in response to any $\mathrm{AM}$ or pulse rate. In response to $\mathrm{AM}$ noise at both $10 \mathrm{~dB}$ and $20 \mathrm{~dB}$ above threshold, the proportion of csz sites showing no synchronization is 0.8; at $30 \mathrm{~dB}$ above threshold, the proportion is 0.71 . In response to pulsed noise, the proportion of csz sites showing no synchronization at any modulation rate is 0.6 and 0.7 at 10 and $20 \mathrm{~dB}$ above threshold, respectively. At $30 \mathrm{~dB}$ above threshold, this proportion falls to 0.3 ; still, the mean cut-off frequency for synchronization in the csz at this level is significantly less than that for Tmc, as shown by the Tukey tests outlined above. In contrast, all sites in the Tmc synchronize to at least one AM or pulse rate at stimulus levels of 20 and 30 $\mathrm{dB}$ above threshold; it is only at $10 \mathrm{~dB}$ above threshold that some Tmc sites show no synchronization $(0.1$ in response to $\mathrm{AM}$ noise, 0.06 in response to pulsed noise).
Outside of the caudal TS, there is also a difference in synchronous activity between more medial and more lateral areas of the TS. Figure 5A plots changes in VS with pulse rate from 26 different sites all located at midlevels of the TS (Fig. 5B). At all sites, VS declines with increasing pulse rate, consistent with the data in Figure 3. We analyzed the pattern of change in VS over sites divided into three groups, defined as follows. The medial-lateral extent of the TS was divided into one-third segments and labeled as medial Tp (the area closest to the midline of the two lobes), medial-lateral $\mathrm{Tp}$, and lateral Tp/Tmc (Fig. 5B). Regression lines describing how VS changes with pulse rate were calculated separately for data in these three groups; these lines are plotted in Figure 5A. VS is highest at the most lateral sites and becomes lower at more medial sites, at any pulse rate. The regression lines for the three groups are clearly separated at low pulse rates; the regression lines for the medial-lateral $\mathrm{Tp}$ and the lateral Tp/Tmc begin to converge at high pulse rates, while that for the medial Tp remains lower. Statistical analyses on these data were performed using multivariate ANOVA; results show a significant effect of site [Hotelling's trace: F $(18,28)=2.37, p=0.02$; observed power $=0.93$ ]. The pattern of differences in VS across sites suggests that there is a lateral-to-medial gradient of synchronous activity in the TS.

\section{Response latency}

Response latency varied considerably in our data, and this variability was related to recording site. The pattern of results with regard to regional differences is the same regardless of which latency measure, mean first spike, or cumulative PST histogram onset, is used; data presented below are based on mean first spike latency. Box plots for the latency data from four different regions in the TS are shown in Figure 6 (left column, AM noise; right column, pulsed noise; note the changes in the $\mathrm{x}$ axis between the individual graphs). For statistical analysis, latency values were log-transformed to achieve homogeneity of variance, and analyzed using one-way ANOVA on each stimulus type and each stimulus level separately. Results of these analyses showed a significant main effect of site for both AM noise and pulsed noise at all stimulus levels tested (see figure caption for F values). Post hoc comparisons using the Tukey HSD test showed that latencies are significantly longer $(p<0.01)$ at csz sites than at both Tmc and Tp sites, for both stimulus types and all stimulus levels. In addition, csz latencies are significantly longer $(p<0.05)$ than $\mathrm{Tl}$ latencies in response to AM noise at all stimulus levels, and in response to pulsed noise at $20 \mathrm{~dB}$ above threshold. There are no significant differences in response latency between $\mathrm{Tl}$ and $\mathrm{Tp}$ at any stimulus level for either stimulus type. 
AM noise
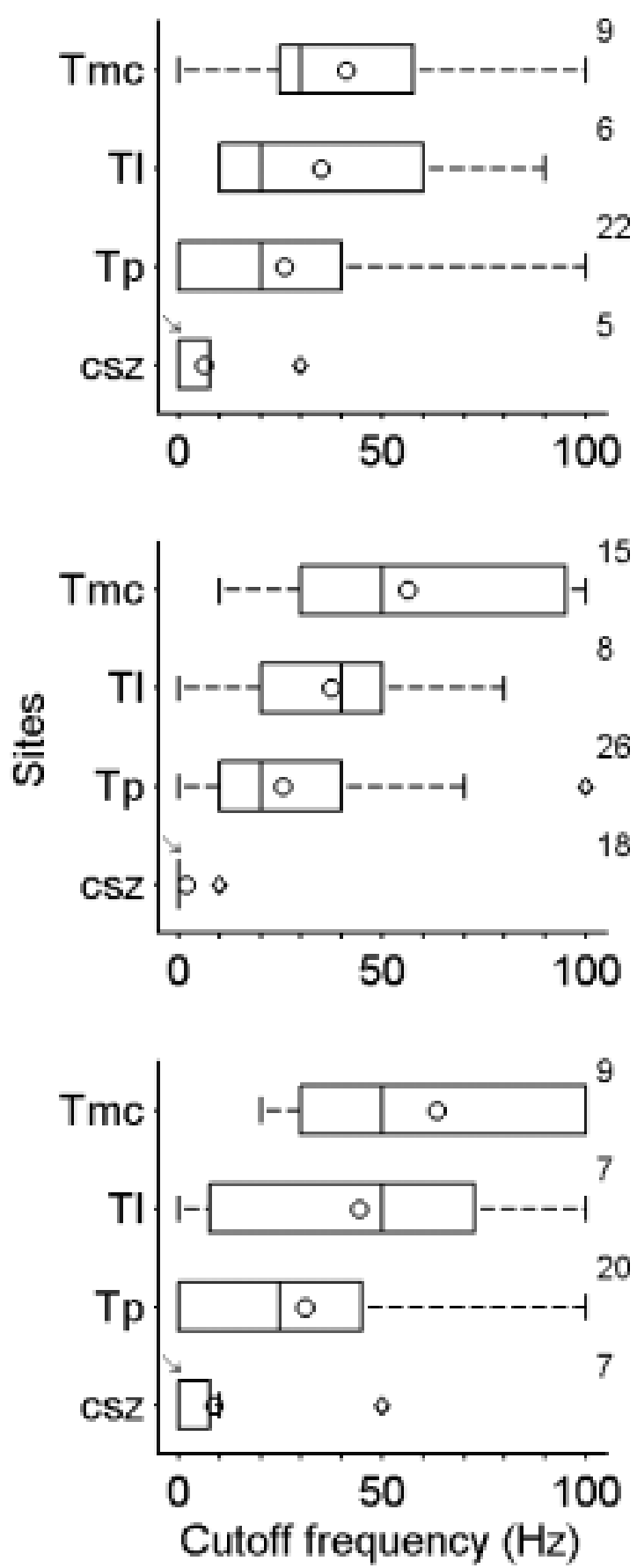

FIG. 4. Cutoff frequencies for significant synchronization in response to AM noise (left column) and pulsed noise (right column) at three different levels above threshold (top row: $+10 \mathrm{~dB}$; middle row: $+20 \mathrm{~dB}$; bottom row, $+30 \mathrm{~dB}$ ). Data are shown separately ( $y$ axis of each plot) as box plots for 4 different areas of the TS (Tmc, $\mathrm{TI}, \mathrm{Tp}, \mathrm{csz}$ ). In each box plot, the center vertical line of the box shows the median; the leftmost and rightmost edges of the box indicate the 25th and 75th percentiles, respectively. The dashed lines extending beyond the box show the remaining data. Outliers, values which fall outside of the dashed lines, are plotted with the diamond symbol (these values were $>1.5$ interquartile range). If the median falls on the edge of the box, an arrow indicates its position. The circle symbol indicates the mean. Data from an individual recording site were included only if at least $3 \mathrm{AM}$ or pulse rates were tested; for this

\section{Pulsed noise}
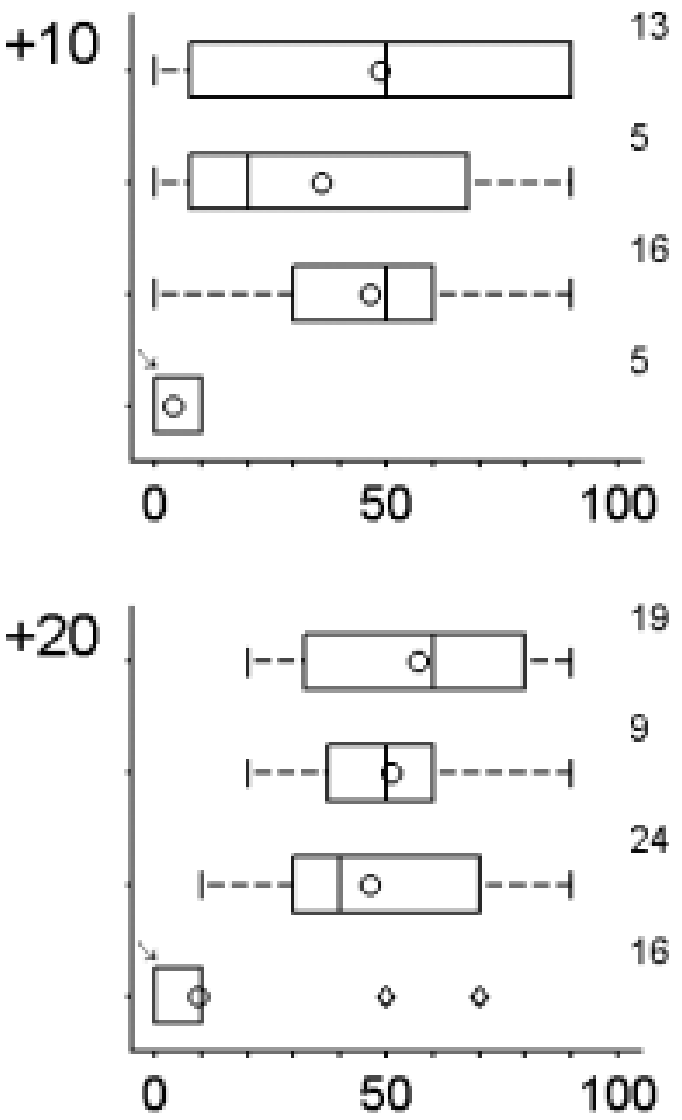

$+30$

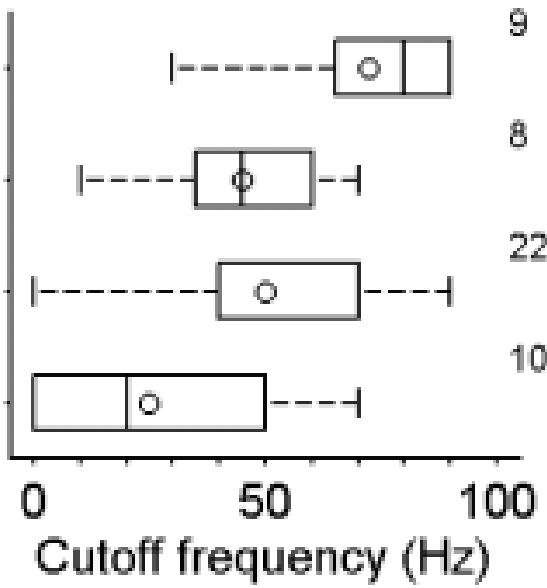

reason, $N$ for $\mathrm{AM}$ noise at +20 and +30 , and pulsed noise at +10 and +20 are smaller than indicated in Table 1 . The $N$ at each individual recording site is indicated by the number at the far right for each site's box plot. One-way ANOVA was performed on the data in each plot, with the null hypothesis being no different in cutoff frequencies between sites. Except for AM noise at $+10 \mathrm{~dB}$, all other ANOVAs yielded a significant main effect of site. For $A M$ noise at $+20 \mathrm{~dB}$, $\mathrm{F}(3,66)=14.01, p<0.001$; for $\mathrm{AM}$ noise at $+30 \mathrm{~dB}, \mathrm{~F}(3,42)=$ 4.13, $p=0.012$. For pulsed noise at $+10 \mathrm{~dB}, \mathrm{~F}(3,38)=2.9, p<$ 0.05; for pulsed noise at $+20 \mathrm{~dB}, \mathrm{~F}(3,67)=13.5, p<0.001$; for pulsed noise at $+30 \mathrm{~dB}, \mathrm{~F}(3,48)=6.1, p=0.001$. Post hoc comparisons with Tukey HSD tests revealed significant differences in cutoff frequencies between Tmc and csz sites (see text for results of other comparisons). 


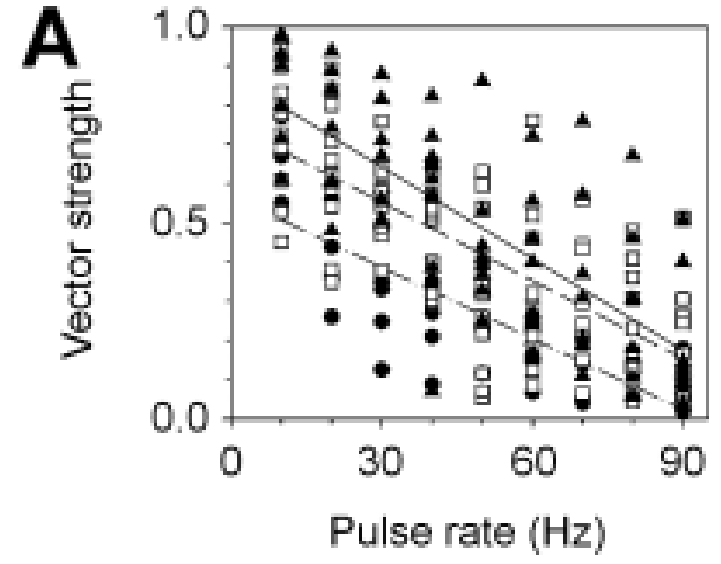

$$
\begin{aligned}
& \text { - ITp/Tmc } \\
& \text { - mITp } \\
& \text { - mTp }
\end{aligned}
$$

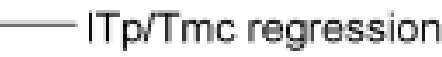
- mITp regression -...- mTp regression

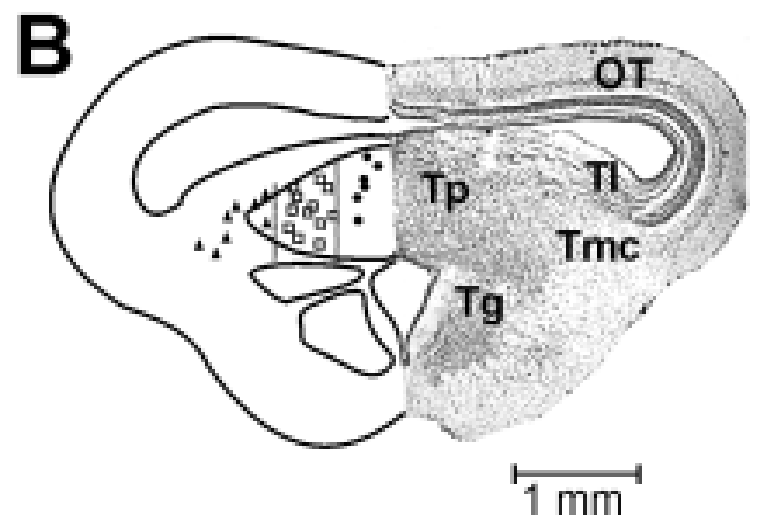

FIG. 5. A Changes in VS over pulse rate for cells located in three different areas of the TS at midlevels. Data were collected at a stimulus level of $20 \mathrm{~dB}$ above threshold. Data for the medial Tp (mTp; $N=$ 4 single unit and 2 multiple unit sites) are shown by the circles; data for the medial-lateral $\mathrm{Tp}(\mathrm{mlTp} ; N=7$ single unit sites and 5 multiple unit sites) are shown by the squares; data for the lateral Tp/Tmc (ITp/ Tmc; $N=7$ single unit sites and 1 multiple unit site) are shown by the triangles. The best-fitting linear regression is shown for each of the 3 areas. For the medial Tp, $y=-0.006 x+.57, r^{2}=0.69$ (dashdot line); for the medial-lateral Tp, $y=-0.0066 x+.75, r^{2}=0.52$ (dashed line); for the lateral Tp/Tmc, $y=-0.0078 x+0.87, r^{2}=0.55$ (solid line). Results of multivariate ANOVA showed a significant main effect of site (see text for details). B Locations of recording sites for data plotted in the top graph as shown on a schematic tracing of the TS. Rostral-caudal position corresponds to level 6 in Fig. 1; actual locations of cells extend up to $250 \mu \mathrm{m}$ rostral to this section. Locations of recording sites from different brains were plotted on the summary schematic using rostral distance from the last appearance of the nucleus isthmus and lateral distance from the midline of the TS as guidelines. The schematic was made and the recording locations from individual brains were plotted by an individual "blind" to the hypothesis being tested.

We explicitly measured spontaneous activity at 53 recording sites. Spontaneous activity ranged from 0 to 45 spikes/s, with a median of 5 spikes/s. Higher levels of spontaneous activity were obtained from multiple unit sites. Over all recording sites tested, level of spontaneous activity varied significantly between different areas of the TS $[\mathrm{F}(3,52)=4.03, p<0.05]$; spontaneous rates were higher in the csz than in Tmc, Tl, or $\mathrm{Tp}$. Because response latencies were also longer in csz, these data suggest that our measures of latency were not appreciably affected by spontaneous activity.

Latency differences are correlated with degree of synchronous activity. For example, for the data plotted in Figure 5, mean latency from medial Tp sites (20 $\mathrm{ms})$ is longer than those from medial-lateral $\mathrm{Tp}$ (16 $\mathrm{ms})$, and lateral $\mathrm{Tp} / \mathrm{Tmc}(14 \mathrm{~ms})$. Over the entire caudal/rostral extent of the TS, the degree of synchronous activity, quantified by cutoff frequency, is negatively correlated with response latency. For AM noise bursts, the correlation between these two parameters is -0.58 ; for pulse trains, the correlation is -0.71 . These correlations are both highly significant $(p<0.001)$.

\section{Population interval coding of period}

Figure 7 shows the pooled ACFs from all sites in Tmc, $\mathrm{Tp}$, and csz in response to pulsed noise presented at 5 rates $(10,30,50,70$, and $90 \mathrm{~Hz})$ at +20 and +30 $\mathrm{dB}$ above threshold (data at $+10 \mathrm{~dB}$ are not shown but are consistent with those shown here). ACFs from the Tmc and Tp show clear interval coding of the period of the stimuli up to rates of $50 \mathrm{~Hz}$, while those from the csz show little such coding even at rates of $10 \mathrm{~Hz}$. This is consistent with the pattern of results seen in individual ACFs (data not shown) and in VS calculated from period histograms (Figure 3). Pooled ACFs from the Tmc and Tp also show peaks at intervals corresponding to multiples of the $70-\mathrm{Hz}$ stimulus period, particularly at levels of $30 \mathrm{~dB}$ above threshold. Moreover, the highest peak in the ACF (excluding the initial peak of the shortest burst-related intervals) is at the stimulus period. Comparison of Figure 7 with Figure 4 shows that the pooled response in the Tmc and $\mathrm{Tp}$ represents the stimulus periodicity at a higher modulation rate than the median synchronized response from individual recording sites. There are no obvious peaks in the ACF from Tp sites in response to $90-\mathrm{Hz}$ pulsed noise at either stimulus level (Fig. 7), while in the Tmc, the ACF continues to show peaks at intervals separated by the stimulus period in response to this stimulus. These data suggest that the pooling of intervals across recording sites improves the detection of the stimulus period in both the Tp and the Tmc, but not in the csz.

We presented a synthetic version of the bullfrog's advertisement call (Fig. 8A, top) to 21 recording sites (14 single unit sites, 7 multiple unit sites) in different parts of the TS, at levels of 10, 20, and $30 \mathrm{~dB}$ above 
AM noise

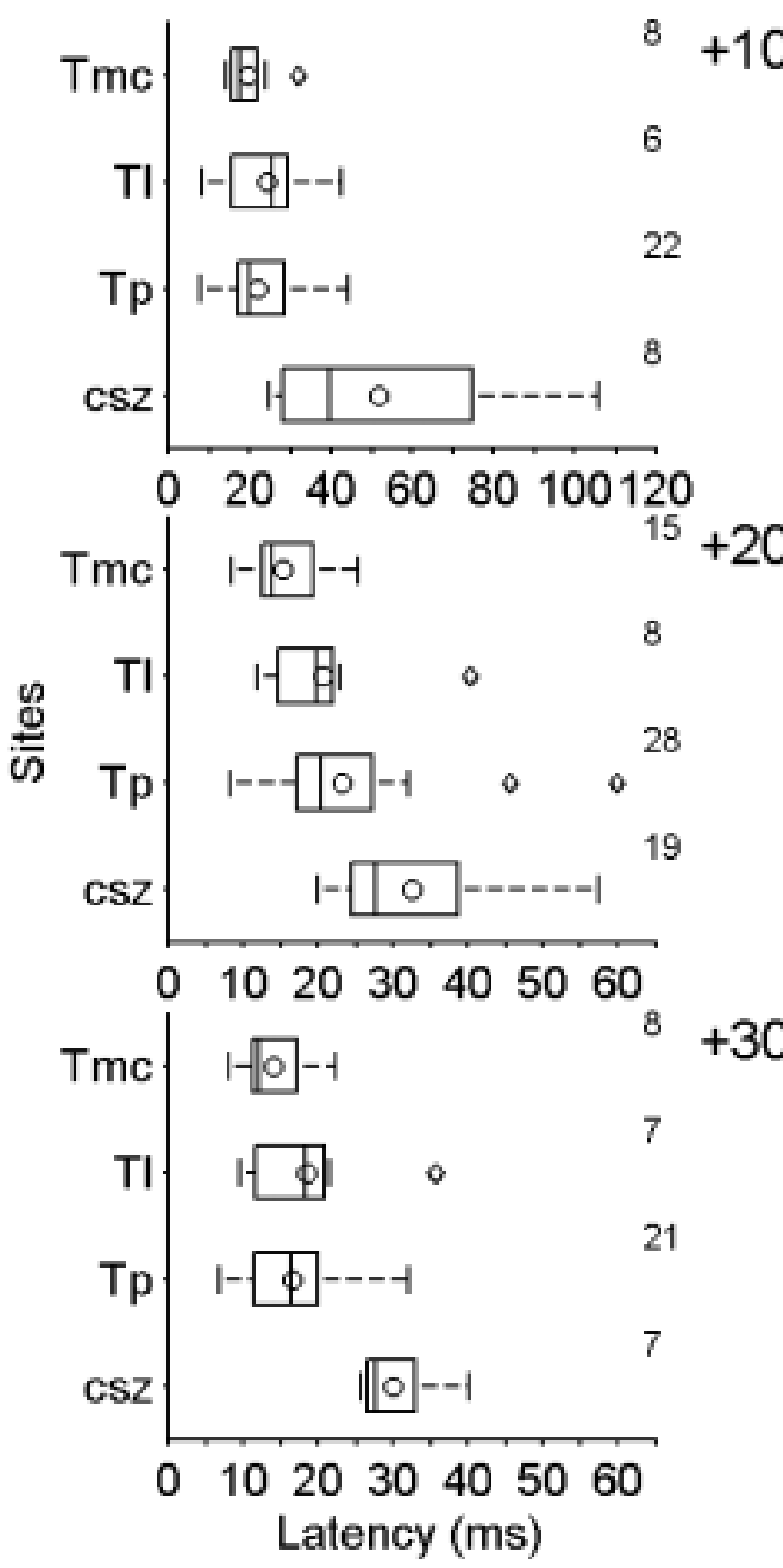

Pulsed noise

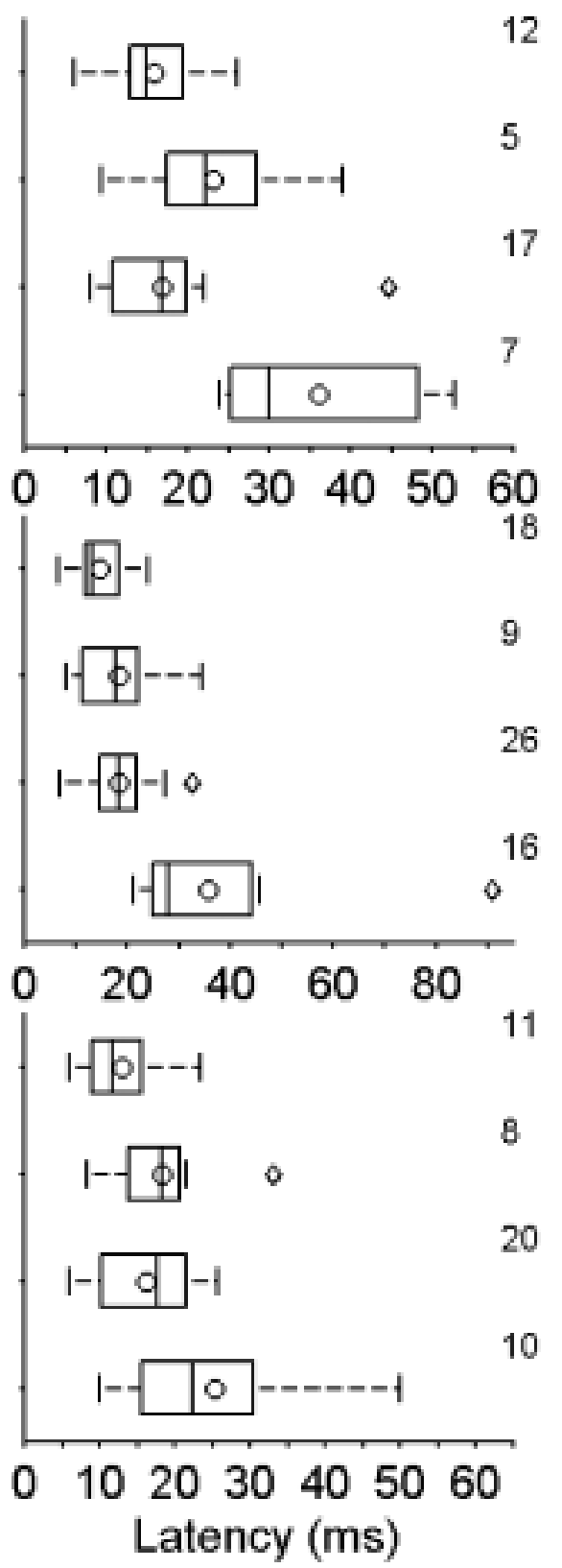

types at all three levels, there was a significant main effect of recording site. For $\mathrm{AM}$ noise at $+10 \mathrm{~dB}, \mathrm{~F}(3,43)=6.5, p=0.001$; for $\mathrm{AM}$ noise at $+20 \mathrm{~dB}, \mathrm{~F}(3,69)=12.8, p<0.001$; for $\mathrm{AM}$ noise at +30 $\mathrm{dB}, \mathrm{F}(3,42)=7.02, p=0.001$. For pulsed noise at $+10 \mathrm{~dB}, \mathrm{~F}(3,40)$ $=7.6, p<0.001$; for pulsed noise at $+20 \mathrm{~dB}, \mathrm{~F}(3,68)=16.3$, $p<0.001$; for pulsed noise at $+30 \mathrm{~dB}, \mathrm{~F}(3,48)=4.13, p=0.011$. Post hoc comparisons with the Tukey HSD test showed that latency differences between csz and Tmc sites were highly significant (see text for details of results of other post hoc comparisons).

at $+10 \mathrm{~dB}(N=5),+20 \mathrm{~dB}(N=10)$, and $+30 \mathrm{~dB}$ $(N=8)$ above threshold clearly reflect the ACF of the stimulus; the plots show peaks in the ACF separated by intervals of $10 \mathrm{~ms}$. At $+30 \mathrm{~dB}$, the highest peak in the ACF is at $10 \mathrm{~ms}(100 \mathrm{~Hz})$, while at $+20 \mathrm{~dB}$, the pooled ACFs of short latency $(<25 \mathrm{~ms})$ responses 


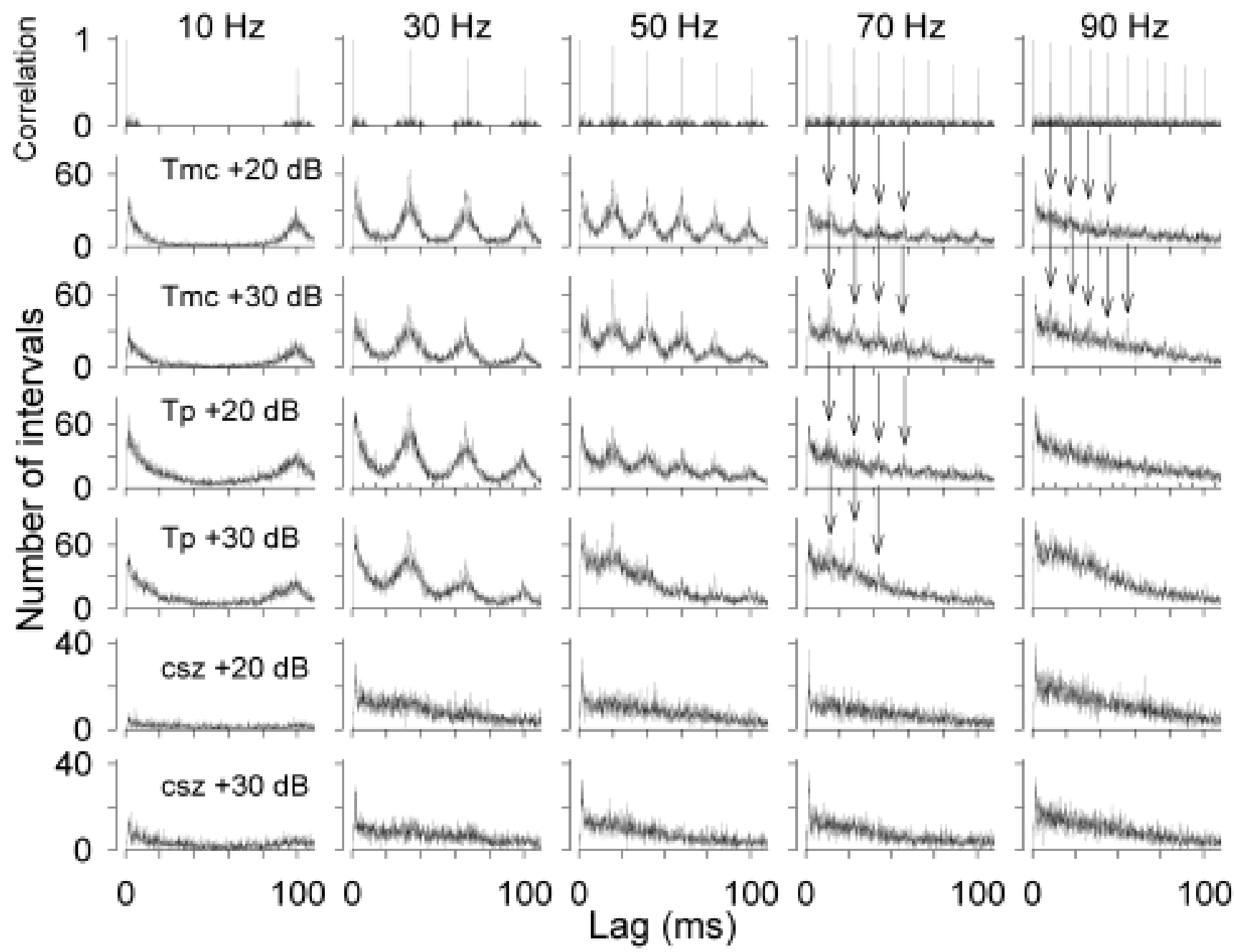

FIG. 7. Pooled all-order interval histograms (ACFs) in response to pulsed noise at 5 different rates $(10,30,50,70,90 \mathrm{~Hz})$. Data were collected at 20 and $30 \mathrm{~dB}$ above threshold. and represent responses from both single unit and multiple unit sites. The top row shows the ACFs of the stimuli. ACFs from the Tmc are shown at $20 \mathrm{~dB}(19$ recording sites) and $30 \mathrm{~dB}$ (11 recording sites) above threshold, as indicated. ACFs from the Tp and TI at mid and rostral levels are

highest peak is at $20 \mathrm{~ms}(50 \mathrm{~Hz})$; still, there are multiple peaks in the ACF separated by a time interval corresponding to the stimulus period. In contrast, the pooled ACFs of long latency ( $>26 \mathrm{~ms}$ ) responses do not show a structure from which the period of the stimulus can be readily extracted at any intensity level $(N=3$ at $+10 \mathrm{~dB} ; N=11$ at $+20 \mathrm{~dB} ; N=10$ at +30 $\mathrm{dB})$. The anatomical locations of the recording sites are shown in Figure 8C. Short latency sites tend to be concentrated in more lateral areas (Tmc, lateral Tp; median latency of $19 \mathrm{~ms}$ ) than long latency sites (median latency of $40 \mathrm{~ms}$ ), which tended to be located more medially ( $\mathrm{Tl}$ and $\mathrm{Tp}$ at midlevels, csz). Moreover, long latency sites also tended to be located more caudally than short latency sites. Although the sample size is smaller than that in the main part of the experiment, combined (at $20 \mathrm{~dB}$ above threshold, 33 recording sites; at $30 \mathrm{~dB}$ above threshold, 28 recording sites). ACFs from the csz are shown in the bottom 2 rows (at $20 \mathrm{~dB}$ above threshold, 16 recording sites; at $30 \mathrm{~dB}$ above threshold, 10 recording sites). Arrows indicate peaks at multiples of the stimulus period for rates of $70 \mathrm{~Hz}$ (Tmc, Tp) and $90 \mathrm{~Hz}(\mathrm{Tmc})$.

the data again clearly show differences in coding of complex signals between lateral and medial regions of the TS.

\section{AM Tuning}

A variety of shapes of rMTFs, including low pass, high pass, band pass, band suppression, and nonselective functions, were observed in the rate responses of both single and multiple units. Figure 9 shows rMTFs from 12 different single unit sites in response to AM noise (rows 1 and 2) and pulsed noise (rows 3 and 4) at 20 and $30 \mathrm{~dB}$ above threshold. These sites were typical of the types of functions observed in the different nuclei of the TS (csz, column A; Tmc, column B; Tp, column C). Band pass (AM tuned) neurons (Fig. 9, 

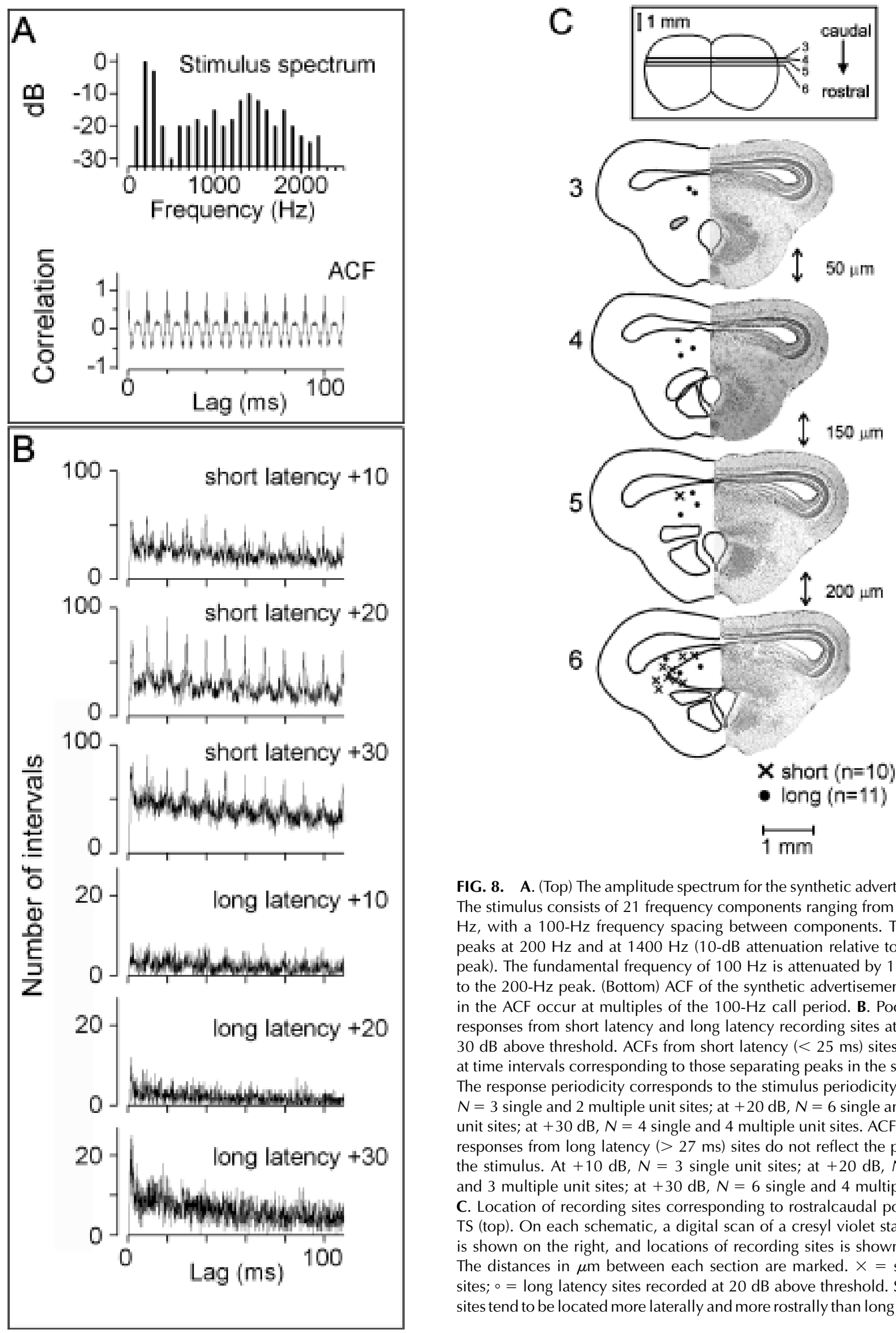

FIG. 8. A. (Top) The amplitude spectrum for the synthetic advertisement call. The stimulus consists of 21 frequency components ranging from 100 to 2200 $\mathrm{Hz}$, with a $100-\mathrm{Hz}$ frequency spacing between components. The spectrum peaks at $200 \mathrm{~Hz}$ and at $1400 \mathrm{~Hz}(10-\mathrm{dB}$ attenuation relative to the $200-\mathrm{Hz}$ peak). The fundamental frequency of $100 \mathrm{~Hz}$ is attenuated by $18 \mathrm{~dB}$ relative to the $200-\mathrm{Hz}$ peak. (Bottom) ACF of the synthetic advertisement call. Peaks in the ACF occur at multiples of the $100-\mathrm{Hz}$ call period. B. Pooled ACFs of responses from short latency and long latency recording sites at 10, 20, and $30 \mathrm{~dB}$ above threshold. ACFs from short latency $(<25 \mathrm{~ms})$ sites show peaks at time intervals corresponding to those separating peaks in the stimulus ACF. The response periodicity corresponds to the stimulus periodicity. At $+10 \mathrm{~dB}$, $N=3$ single and 2 multiple unit sites; at $+20 \mathrm{~dB}, N=6$ single and 4 multiple unit sites; at $+30 \mathrm{~dB}, N=4$ single and 4 multiple unit sites. ACFs of summed responses from long latency ( $>27 \mathrm{~ms}$ ) sites do not reflect the periodicity of the stimulus. At $+10 \mathrm{~dB}, N=3$ single unit sites; at $+20 \mathrm{~dB}, N=8$ single and 3 multiple unit sites; at $+30 \mathrm{~dB}, N=6$ single and 4 multiple unit sites. C. Location of recording sites corresponding to rostralcaudal position in the TS (top). On each schematic, a digital scan of a cresyl violet stained section is shown on the right, and locations of recording sites is shown on the left. The distances in $\mu \mathrm{m}$ between each section are marked. $\times=$ short latency sites; $\circ=$ long latency sites recorded at $20 \mathrm{~dB}$ above threshold. Short latency sites tend to be located more laterally and more rostrally than long latency sites. 


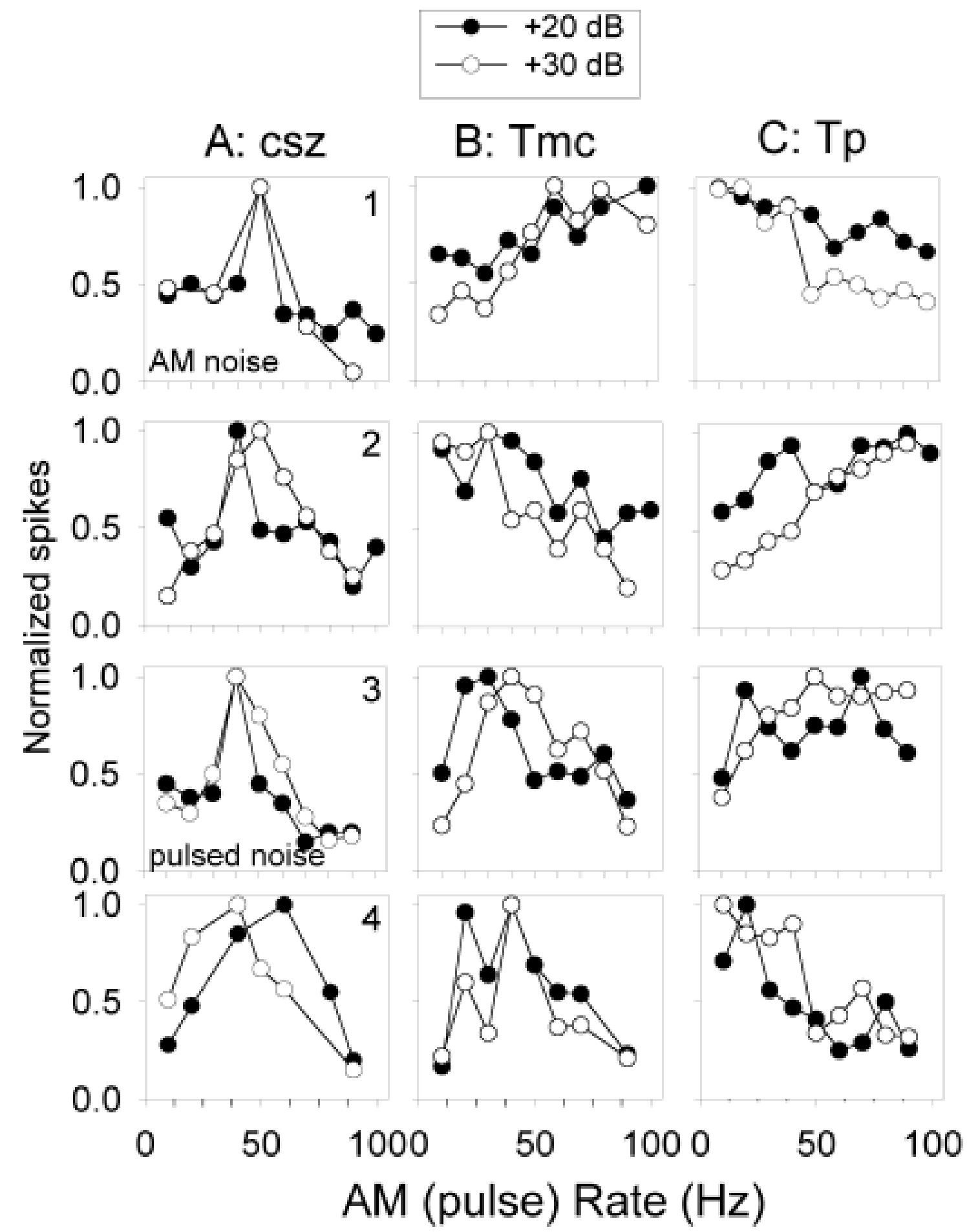

FIG. 9. Rate modulation transfer functions (rMTF) plotting number of spikes against AM rate (rows 1 and 2) and pulsed noise rate (rows 3 and 4) for 12 single unit recording sites in the TS. Data collected at $20 \mathrm{~dB}$ above threshold are indicated by closed circles, and data collected at $30 \mathrm{~dB}$ above threshold are indicated by open circles (see legend). In each plot, number of spikes is normalized to the maximum number observed in response to that stimulus type. The shapes of the rMTFs in column A meet the criteria for bandpass rMTFs as classified by Rose and Capranica (1985). For the function in A2, at
$20 \mathrm{~dB}$ above threshold, spike count is at $55 \%$ of the maximum at $10 \mathrm{~Hz}$, slightly above the $50 \%$ criterion. These recording sites were located in the csz (see Fig. 10 for exact locations). Recording sites shown in column B are located in Tmc, and recording sites in column $\mathrm{C}$ are located in $\mathrm{Tp}$ at midlevels. Low-pass rMTFs are shown in B2, $\mathrm{C} 1$ and $\mathrm{C} 4$; high pass rMTFs are shown in B1 and C2. C2 shows both a high-pass function (at $30 \mathrm{~dB}$ above threshold), and a multipeaked, nonselective function (at $20 \mathrm{~dB}$ above threshold). 
column A) show maximal responses to AM or pulse rates between 20 and $80 \mathrm{~Hz}$, with spike count dropping to at least $50 \%$ of the maximum at lower and higher rates (for the function in $\mathrm{A} 2$, at $20 \mathrm{~dB}$ above threshold, spike count at $10 \mathrm{~Hz}$ is at $55 \%$ of maximum). Synchronized activity was low at these recording sites. Other cells tested at AM rates as high as $200 \mathrm{~Hz}$ (data not shown) showed peaks in the rMTF at rates of $100 \mathrm{~Hz}$. Note that stimulus level does not appreciably affect the overall shape of the rMTF, but in some cases (A $2,4)$ produces a shift in the peak of the function to another AM or pulse rate. These recordings were obtained from sites in the medial portions of the caudal TS, from the level of the nucleus isthmus, up to approximately $200 \mu \mathrm{m}$ rostral to the disappearance of this nucleus (Fig. 10). Within this caudal area, band pass-tuned neurons are located in the more cell dense layers ventral to the optic ventricle, comprising caudal areas of $\mathrm{Tl}$ and in the more ventral cell sparse regions closer to the nucleus isthmus (in the caudal-most regions) or to the ventral tegmentum (in slightly more rostral regions). Sites showing lowpass, high-pass, and band-suppression characteristics were also observed in the csz for some stimulus types and levels (data not shown).

rMTFs from recording sites in the Tmc are shown in Figure 9, column B. In response to AM noise, Tmc sites in our sample show responses that were primarily high pass $(\mathrm{B} 1,+30 \mathrm{~dB})$, low pass $(\mathrm{B} 2,+30 \mathrm{~dB})$, or nonselective (data not shown) in shape. No clear instances of band pass tuning were observed at Tmc sites in response to AM noise. In response to pulsed noise, some rMTFs from single unit sites in the Tmc (B3) could be characterized as band pass; note, however, that the peak in the function shifts depending on stimulus level. The rMTF shown in B4 has a multipeaked shape at both stimulus levels, but with the same peak in response (at $50 \mathrm{~Hz}$ ). High pass, low pass, and nonselective rMTFs were also observed in response to pulsed noise (data not shown). The locations of band pass rMTFs in the Tmc are shown in Figure 10. Most of the band pass neurons are located in caudal regions of the Tmc (comprising the lateral portions of the ventral toral zone of Wilczynski 1988), but are also found up to midlevels of the TS.

rMTFs shown in Figure 9, column $\mathrm{C}$ were recorded from cell dense regions of the $\mathrm{Tp}$ or the $\mathrm{Tl}$, at midlevels of the TS, in the areas corresponding to the onion skin layers described by Feng (1983), or at more rostral levels where the optic ventricle and the third ventricle merge. We found no clear evidence of band pass responses in the $\mathrm{Tp}$ or the $\mathrm{Tl}$ at these levels. Typically, rMTFs are low pass $(\mathrm{C} 1, \mathrm{C} 4)$, high pass (C2), or nonselective (C3) in shape. In some cases (C1, C3), stimulus level changes the shape of the rMTF, while in other
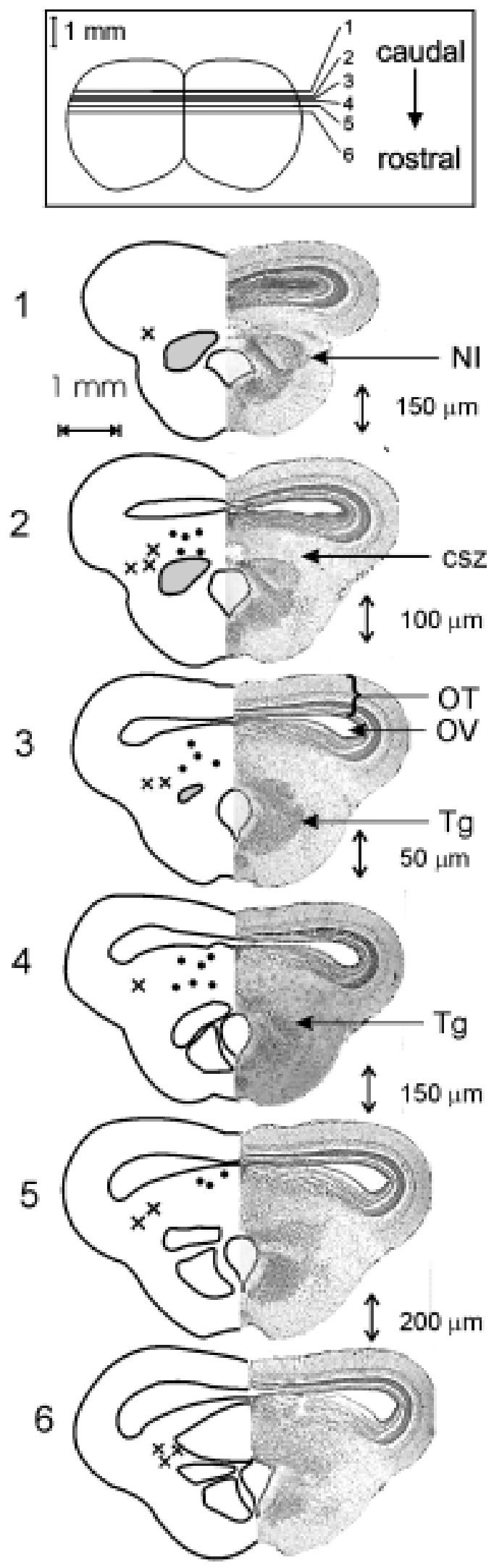
cases $(\mathrm{C} 2, \mathrm{C} 4)$, the shape of the rMTF is similar at the two intensity levels.

A direct comparison of rMTFs from the same sites in response to $\mathrm{AM}$ and pulsed noise (both at $20 \mathrm{~dB}$ above response threshold for that stimulus type) is shown in Figure 11. All of the data are from single unit recordings. The rMTF in Figure 11A shows a band pass shape in response to AM noise that becomes multipeaked in shape in response to pulsed noise. Peak spike count remains at $50 \mathrm{~Hz}$ in both rMTFs, however. Figure 11B shows a band pass rMTF to AM noise that becomes low pass in shape to pulsed noise, with a shift in the peak of the function to a higher pulse rate. Figure 11C shows a low-pass response to AM noise that becomes a high-pass response to pulsed noise; although both functions show a dip in responding at intermediate rates, the location of the largest dip in spike count varies. Because spike count does not increase above $50 \%$ at both extremes of the function, these rMTFs do not meet the criterion for band-suppression functions.

\section{DISCUSSION}

Different subnuclei within the bullfrog's TS represent acoustic features related to waveform periodicity by different neural codes. Long latency cells located in the caudal/medial (csz) portions of the TS encode stimulus period by a rate code. AM-tuned neurons are found most often in these areas of the TS. Some cells in caudal up to midlevels of the Tmc show band pass responses to pulsed noise, but not to AM noise. Short latency cells located laterally ( $\mathrm{Tmc}$, lateral $\mathrm{Tp}$ ) show a broader frequency range of synchronous activity than cells in the csz, and represent stimulus period by a time interval code. Pooling of all-order interval histograms across short latency cells to produce a population response extends the range of temporally-based activity over what is typically observed in most single cell responses. These data suggest that lateral (lateral Tp, Tmc) and medial (csz) areas of the TS play different roles in coding of complex sounds; specifically, csz cells reflect the time-to-place transformation described previously (reviewed in Langner 1992), while lateral

FIG. 10. Locations of bandpass neurons. Levels of sections are referred to the top schematic. The distances in $\mu \mathrm{m}$ between each section are marked. Bandpass neurons in the csz, including those whose data are shown in Figure 9, are indicated by dark circles. Band pass sites in more lateral (Tmc) areas, at both caudal and rostral levels, are indicated by crosses. There are significant differences in response latencies between the more medial csz sites and the more lateral Tmc sites (see Results). Data are based on responses of 30 cells stimulated at $20 \mathrm{~dB}$ above threshold.
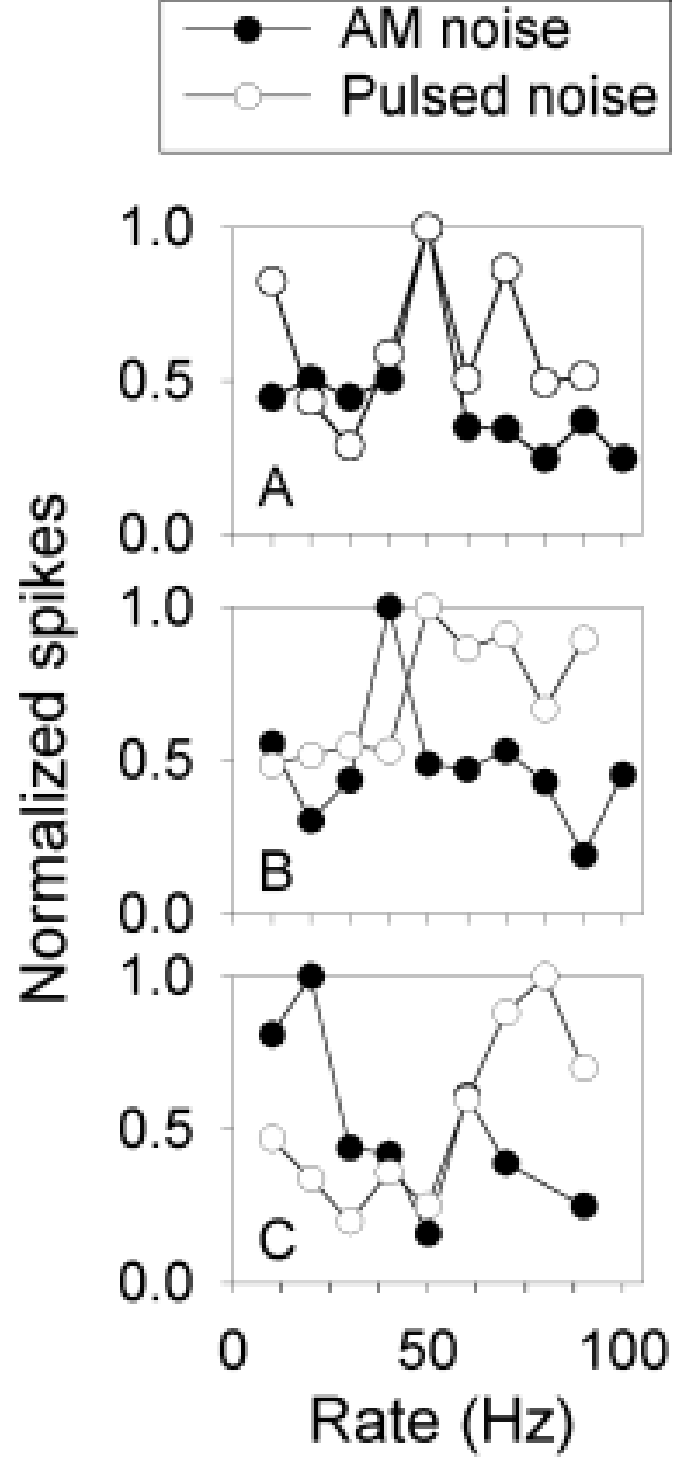

FIG. 11. The rMTF can change in shape with changes in stimulus type. A, csz, single unit recording; bandpass rMTF in response to AM noise shows several peaks in response to pulsed noise, but the rate eliciting maximal spikes remains the same. $B$, csz, single unit recording; bandpass rMTF in response to AM noise becomes highpass in response to pulsed noise, and the peak of the function shifts. C, Tp, single unit recording; low-pass rMTF to AM noise becomes high-pass in response to pulsed noises. Although both rMTFs show dips in responding at intermediate rates, they do not reach the criterion for band suppression functions and the location of the maximum dip differs.

Tp and Tmc cells code stimulus period by time interval responses. These functional differences between TS subdivisions may reflect different patterns of afferent input to the midbrain from different functional and morphological cell types in the medullary auditory nucleus (dorsal lateral nucleus, DLN, in anurans, cochlear nucleus in mammals; Kim et al. 1990; Rhode 
and Greenberg 1994; Feng and Lin 1994), where segregation of temporal and rate codes are also evident.

\section{Temporal coding of period}

Our data confirm previous reports (e.g., Rose and Capranica 1985; Epping and Eggermont 1986a,b; Gooler and Feng 1992; Bibikov and Nizamov 1996; reviews: Simmons and Buxbaum 1996, Feng and Schellart 1999) that TS cells have a much more restricted range of cycle-by-cycle phase-locked responding than do eighth nerve fibers. Specifically, in the bullfrog, eighth nerve fibers synchronize to the envelopes of complex signals up to modulation rates of $800 \mathrm{~Hz}$ (Simmons and Buxbaum 1996). Coding for the period of complex signals in the bullfrog's eighth nerve is achieved by a temporal mechanism based on phaselocked discharges or interspike interval measurements (Schwartz and Simmons 1990). Individual fibers have broad spectral tuning, and their synchronized discharges are captured by the envelope of multiple harmonic signals, regardless of characteristic frequency (Simmons and Ferragamo 1993; Simmons et al. 1993). Some neurons in the frog's DLN can synchronize to the envelopes of AM tones up to modulation rates of $300 \mathrm{~Hz}$, but there are considerable differences in the extent of phase-locked activity between different functional classes of DLN cells (Feng and Lin 1994). Thus, a transformation and a functional segregation of the neural code first appears in the medulla, at least for some cell types, and is further reflected in the midbrain.

In our data, neither single nor multiple unit activity in any region of the TS show significant synchronization to the envelopes of either AM noise or pulsed noise at modulation rates beyond $100 \mathrm{~Hz}$; however, within this limited, low modulation range, there are significant differences in the extent of phase-locked responding in different TS subnuclei. Arrays of phaselocked activity from the eighth nerve, and presumably also from the medulla, are clearly represented in neural responses recorded from lateral regions of the TS (Tmc, lateral Tp). Recording sites in the Tmc typically show a much broader range of phase-locked response than those in the csz, where some cells do not phase lock to modulation rates even as low as $10 \mathrm{~Hz}$ (Figs. 3 and 4). Regional differences in synchronous responding extend up to mid and rostral levels of the TS (Fig. 5), and we suggest that there is a lateral-tomedial gradient of phase-locked activity in the TS. We hypothesize that this lateral-to-medial gradient reflects different patterns of afferent input from medullary auditory nuclei.

The median frequency cutoff for synchronization is higher in response to pulsed noise than in response to AM noise (Fig. 4). Although both AM and pulsed noise convey a percept of pitch to humans (Flanagan and Gutmann 1960; Burns and Viemeister 1976), the salience of the perceived pitch differs (Plomp 1976). This difference in salience might be reflected in the differences in the absolute levels of synchronous activity evoked by these different types of stimuli; however, it is not known if AM noise and pulsed noise with the same envelope modulation evoke different perceptual saliences to bullfrogs. The AM noise and pulsed noise used in this study differed in both duration and in rise time, and differences in synchronous activity to these two stimulus types might reflect these differences in stimulus parameters. Duration and rise time influence TS response properties in other species of frogs (Gooler and Feng 1992; Diekamp and Gerhardt 1995).

Our data also show that a neural code based on calculation of time intervals between spikes can reflect the period of complex stimuli on a population level (Figs. 7 and 8B). Waveform periodicity of AM noise, pulsed noise, and a complex multiple harmonic signal is represented in the pooled all-order interspike interval distributions (ACFs) of responses of short latency cells in the lateral areas of the TS. The population code extends the range of synchronous activity in the lateral TS to frequencies higher than those typically encoded by most single cells (Fig. 4). Pooling of responses from groups of cells, which individually might look like poor phase-locked responders, can give rise to a more precise and statistically significant representation, by virtue of the increased spike numbers resulting from pooling. In effect, pooling uncovers the underlying temporal sensitivity of these neurons. Note, however, that even pooled ACFs summed over all recording sites in the csz do not reflect waveform periodicity. This shows that there are fundamental differences in signal processing between these different areas of the TS.

Peaks in the ACF of the bullfrog's advertisement call (Fig. 8A) occur at the same time intervals as peaks in the population ACF from short latency sites in the Tmc and lateral Tp (Fig. 8B). This population coding remains robust with changes in stimulus level, over the $30 \mathrm{~dB}$ range of levels presented here. Simmons and Bean (2000) have shown that male bullfrogs can behaviorally detect the presence of single mistuned harmonics in a synthetic advertisement call over an intensity range of $30 \mathrm{~dB}$ (from 50 to $80 \mathrm{~dB} \mathrm{SPL}$ ), as long as the mistuning does not alter the stimulus envelope. In their data, stimulus level had no significant influence on the animals' ability to discriminate differences in envelope. This suggests that bullfrogs' perception of periodicity is invariant with changes in level, consistent with the physiological data presented here, and with human psychoacoustic data on the level-independence of judgments of the pitch of complex harmonic sounds (Plomp 1976). 
The bullfrog's advertisement call has a first harmonic period of $100 \mathrm{~Hz}$ (Fig. 8A); in some cases, males add extra modulation of $20-30 \mathrm{~Hz}$ to the envelope of the signals, but they do not use signals with modulation rates higher than $150 \mathrm{~Hz}$ (personal observations). Moreover, males respond less strongly to synthetic advertisement calls with modulation rates of $200 \mathrm{~Hz}$ than to those with modulation rates of $100 \mathrm{~Hz}$ (Hainfeld et al. 1996). Our data, therefore, show that a time interval code in the lateral TS can extract the period of complex signals over a modulation range that is biologically significant to the animal.

\section{Response latency}

There were significant differences in response latency between recording sites in different subnuclei of the TS (Fig. 6). In particular, cells in the csz had significantly longer latencies than those in the Tmc. Short latency cells were not restricted to the Tmc, but were also found in both the lateral Tp and Tl (Fig. 6); one Tmc cell had a latency as long as $29.5 \mathrm{~ms}$ (population medians ranged from 12 to $16.25 \mathrm{~ms}$, depending on stimulus type and level). The range of latencies recorded in this study, in both lateral and medial areas of the TS, are within the range of latencies observed in intracellular recordings from isolated frog midbrain preparations (Luksch and Walkowiak 1998). This suggests that short latency responses in our sample cannot be attributed solely to recordings from incoming lateral lemniscal fibers, although we cannot exclude the possibility that some of our recordings might reflect fiber activity rather than somal activity. Lateral lemniscal fibers course laterally-to-medially to their termination sites in the TS, but are typically located more ventral than our recording sites (Wilczynski 1988). Long latencies recorded here are at the low end of the range reported by Alder and Rose (1998) for AMtuned neurons in the TS (exact recording sites not known). Animals in that study were maintained at a lower body temperature than those in this study, and this factor might have influenced response latencies.

Both short and long latency responses were observed in both single unit and multiple unit recordings, and were recorded with both tungsten microelectrodes and HRP-filled glass micropipettes, suggesting that the latency variability we observed is not an artifact of technique.

\section{Rate coding of period}

Our data confirm that AM tuned (band pass) neurons are present in the frog's TS, and that these neurons code stimulus periodicity by a rate rather than a time code. Band pass neurons comprise a relatively small proportion of our total sample of recording sites (between about 12 and 25\%, depending on stimulus type and level). This proportion is similar to that reported in other studies in the frog TS (e.g., Rose and Capranica 1985; Epping and Eggermont 1986a,b; Gooler and Feng 1992; Diekamp and Gerhardt 1995). It is important to note, however, that some rMTFs classified as high-pass might instead be band pass neurons tuned to high modulation rates $(>100 \mathrm{~Hz})$; this cannot be excluded in our data because we restricted $\mathrm{AM}$ rates tested to those with biological relevance to the bullfrog. We presented stimuli with AM rates as high as $200 \mathrm{~Hz}$ to a small subset of cells in the csz and were unable to find band pass rMTFs tuned to rates above $100 \mathrm{~Hz}$; however, the small sample size precludes firm conclusions.

In other species of anurans, peaks in the rMTF have been observed at the envelope period of the species advertisement call (Rose and Capranica 1985; Diekamp and Gerhardt 1995; reviewed in Feng and Schellart 1999). We observed rMTFs from cells in the csz with peaks ranging from 10 to $100 \mathrm{~Hz}$, which spans the bullfrog's range of behavioral sensitivity to modulation (Hainfeld et al. 1996), but we did not find band pass neurons with peaks restricted only to the envelope period of the advertisement call.

Our data extend these previous reports of AM tuning in the frog's TS (reviews: Simmons and Buxbaum 1996; Feng and Schellart 1999) by showing that band pass neurons are concentrated in caudal areas of the TS, both medially (csz) and more laterally (Tmc or lateral part of the ventral toral zone as described by Wilczynski 1988). This is consistent with data of Rose and Capranica (1985). Within the csz and caudal Tmc, we found no systematic topographical arrangement of cells tuned to particular AM rates, as observed in the cat by Schreiner and Langner (1988). Because we pooled recording locations across animals to make a composite representation, we cannot conclude that no such topographical arrangement exists in the bullfrog, only that it was not obvious in our data. Our data do show that band pass neurons are most commonly found in a spatially-restricted area of the TS, and future studies can take advantage of this finding to search for a "periodotopic" map. It is also interesting to note that band pass neurons are concentrated outside the tonotopically-organized, cell dense area of the $\mathrm{Tp}$ as defined by Feng (1983), although we did not systematically record BFs of single unit sites. This again suggests that functional differentiation exists within the TS.

Consistent with previous data (Rees and Moller 1987; Eggermont 1990; Gooler and Feng 1992; Diekamp and Gerhardt 1995; but see Rose and Capranica 1985), the tuning of band pass neurons can vary with stimulus type and intensity. It is not clear how a ratebased model of pitch extraction accounts for these changes, or for differences in the salience of pitches 
evoked by different kinds of complex signals (Plomp 1976). The shifts in the peak AM sensitivity shown by some of our cells with changes in stimulus type are greater than what would be expected in psychoacoustic pitch shifts reported by human listeners (Plomp 1976). Although bullfrogs are sensitive to shifts in the waveform periodicity of complex signals (Hainfeld et al. 1996; Simmons and Bean 2000), as discussed above, it is not known whether they perceive shifts in pitch between AM noise and pulsed noise. Male green tree frogs show similar patterns of behavioral sensitivity to $\mathrm{AM}$ tones and $\mathrm{AM}$ noise differing in modulation rate (Allan and Simmons 1994), suggesting that it is the period of the signal, and not its spectral structure, that guides behavior in this species. We also show that the peak rate in some rMTFs in the csz varies with stimulus level (Fig. 9). There is no obvious behavioral correlate to this effect; indeed, signal level does not seem to affect the ability of male bullfrogs to detect the periodicity of a synthetic advertisement call (Simmons and Bean 2000). The invariance of a percept of periodicity with level would presumably aid a vocalizing male or approaching female frog to discriminate between a loud call from a heterospecific male and a soft call from a conspecific male.

\section{Circuits for representation of periodicity}

Both spatial and temporal models for encoding pitch in the central auditory system have been described (reviewed in Lyon and Shamma 1996), but there is no clear agreement on which type of model best explains both neurophysiological and behavioral data. This lack of agreement is based in large part on a relative paucity of neurophysiological data from central auditory nuclei collected specifically in relation to neural computations underlying pitch. Moreover, there are few behavioral data available from animal subjects paralleling physiological data collected from the same species. Pitch coding based on autocorrelation-like analysis was originally proposed by Licklider (1951). This model uses a series of delay lines based on axonal length to detect a correlation between a stimulus input and a neuronally-delayed copy of itself. This allows the timing of neural discharges making up one stream of activity from the cochlea to be compared centrally with other streams to detect consistent patterns or periods. The output of these delay lines then feed into a series of coincidence detectors, which represent the common time intervals or periods between arrays of incoming phase-locked discharges. Langner (1983, 1992) extended this model by incorporating neurons with intrinsic oscillations (oscillation frequencies independent of stimulus period) which serve as an internal reference for pitch measurements. The output of these oscillator neurons, along with the output of "integrator" neurons, feed into a series of coincidence detecting (AM tuned) neurons, where the final pitch output is computed.

Representation of pitch from interval measurements on incoming arrays of neural activity requires a series of delay lines (Lyon and Shamma 1996) or intrinsic oscillators (Langner 1983). An anatomical substrate for delay lines has not yet been observed in the frog auditory brainstem, although the shifts in latency with stimulus type and recording area observed here suggests that delay lines may be present. Although we observed some PST patterns in the Tmc and lateral Tp showing chopper-type responses (data not shown), our data are currently insufficient to determine whether these neurons act like the oscillator neurons described by Langner (1983). Band pass neurons may act as coincidence detectors for shared periodicity, although, as discussed above, the observations that these neurons change their peak sensitivity with stimulus type and level are problematic for a pitch extraction model.

Our data suggest that there may be parallel streams for processing of spectral and temporal acoustic features related to periodicity in the TS. Such a kind of parallel processing for time and frequency coding was described by Hall and Feng (1987) for the frog's auditory thalamus, and parallel pathways for processing of acoustic features related to sound localization have been described in the brainstem of other vertebrates (reviewed in Carr 1993). HRP labeling studies show that Tmc and Tp receive different patterns of input from auditory nuclei in the medulla (DLN and superior olive) and project to different areas of the thalamus (Hall and Feng 1987; Feng and Lin 1991). It is not known whether different morphological or functional cell types in the DLN (Feng and Lin 1994) project to different functional areas of the TS, but our data suggest that this is indeed the case. For example, the Tmc might receive input from those DLN cells showing good phase locked activity, while the Tp might receive input from DLN cells showing poorer phase locking. This has not been systematically examined, either on an anatomical or on a physiological level. Latency differences between lateral and medial TS might arise from differences in lengths of input fibers, or via intermediate synapses in another nucleus (the superficial reticular nucleus, for example). It is possible that the synchronous, short latency cells in the Tmc feed into the longer latency, band pass cells in the csz, although the large differences in response latency between cells in these different areas argues against direct input. The csz might also integrate activity from other areas in the TS, and from other auditory regions in the brain, such as the thalamus. An investigation of the specific connectivity of the physiologically-distinct 
areas we have identified in the TS to other central auditory nuclei would be useful in devising an overall model of periodicity extraction in the frog's central auditory system.

\section{ACKNOWLEDGMENTS}

We thank Kyler Eastman, Maria Molina, and Jason Ruggiero for assistance. Seth Horowitz prepared the anatomical figures. This research was supported by a grant from the National Institute of Neurological Diseases and Stroke (R01NS28565 to AMS).

\section{REFERENCES}

Alder TB, Rose GJ. Long-term temporal integration in the anuran auditory system. Nature Neurosci. 1:519-523, 1998.

ALLAn SE, Simmons AM. Temporal features mediating call recognition in the green treefrog, Hyla cinerea: Amplitude modulation. Anim. Behav. 47:1073-1086, 1994.

Batschelet E. Circular Statistics in Biology. Academic Press, New York, 1981.

BiBikov NG, NizAmov SV. Temporal coding of low-frequency amplitude modulation in the torus semicircularis of the grass frog. Hear Res. 101:23-44, 1996.

BODNAR D. The separate and combined effects of harmonic structure, phase, and FM on female preferences in the barking treefrog (Hyla gratiosa). J. Comp. Physiol. A 178:173-182, 1996.

Burns EM, Viemeister NF. Nonspectral pitch. J. Acoust. Soc. Am. 60:863-869, 1976.

CARIANI PA. Neural timing nets for auditory computation. GREenberg S, SLANEy M. Computational Models of Auditory Function. IOS Press, New York, 1999, in press.

Cariani PA, Delgutte B. Neural correlates of the pitch of complex tones. I. Pitch and pitch salience. J. Neurophysiol. 76:16981716, 1996a.

Cariani PA, Delgutte B. Neural correlates of the pitch of complex tones. II. Pitch shift, pitch ambiguity, phase invariance, pitch circularity, rate pitch, and the dominance region for pitch. J. Neurophysiol. 76:1717-1734, 1996b.

CARR CE. Processing of temporal information in the brain. Ann. Rev. Neurosci. 16:223-243, 1993.

Diekamp B, Gerhardt HC. Selective phonotaxis to advertisement calls in the gray treefrog Hyla versicolor: behavioral experiments and neurophysiological correlates. J. Comp. Physiol. A 177:173$190,1995$.

EgGERMONT J. Temporal modulation transfer functions for single neurons in the auditory midbrain of the leopard frog. Intensity and carrier-frequency dependence. Hear Res. 43:181-198, 1990.

EPPING WJM, EgGERMONT JJ. Sensitivity of neurons in the auditory midbrain of the grassfrog to temporal characteristics of sounds. I. Stimulation with acoustic clicks. Hear Res. 24:37-54, 1986a.

EPPING WJM, EgGERMONT JJ, Sensitivity of neurons in the auditory midbrain of the grassfrog to temporal characteristics of sounds. II. Stimulation with amplitude modulated sound. Hear Res. 24:55$72,1986 \mathrm{~b}$

Evans EF. Pitch and cochlear nerve fiber temporal discharge patterns. Klinke R, Hartmann R Hearing: Physiological Bases and Psychophysics. Springer-Verlag, New York, 1983, 140-146.
FAY RR. Psychophysics and neurophysiology of temporal factors in hearing by the goldfish: Amplitude modulation detection. J. Neurophysiol. 44:312-332, 1980.

FAY RR, SIMMONS AM. The sense of hearing of fishes and amphibians. FAY RR, POPPER AN. Comparative Hearing: Fish and Amphibians. Springer-Verlag, New York, 1999, 269-318.

FENG AS. Morphology of neurons in the torus semicircularis of the northern leopard frog, Rana pipiens pipiens. J. Morphol. 175:253$269,1983$.

FENG AS, LIN W-Y. Differential innervation patterns of three divisions of frog auditory midbrain (torus semicircularis). J. Comp. Neurol. 306:613-630, 1991.

Feng AS, Lin W-Y. Phase-locked response characteristics of single neurons in the frog "cochlear nucleus" to steady-state and sinusoidally-amplitude-modulated tones. J. Neurophysiol. 72:22092221, 1994.

Feng AS, Schellart NAM, Central auditory processing in fishes and amphibians. FAY RR, POPPER AN. Comparative Hearing: Fish and Amphibians. Springer-Verlag, New York, 1999, 218-268.

Ferragamo MJ, Haresign T, Simmons JA. Frequency tuning, latencies, and responses to frequency-modulated sweeps in the inferior colliculus of the echolocating bat, Eptesicus fuscus. J. Comp. Physiol. A 182:65-79, 1998.

Flanagan JL, Gutman N. On the pitch of periodic pulses. J. Acoust. Soc. Am. 32:1308-1319, 1960.

GERHARDT HC. Mating call recognition in the green treefrog ( Hyla cinerea): significance of some fine-temporal properties. J. Exp. Biol. 74:59-73, 1978.

Gooler DM, Feng AS. Temporal coding in the frog auditory midbrain: The influence of duration and rise-fall time on the processing of complex amplitude-modulated stimuli. J. Neurophysiol. 67:1-21, 1992

HAINFELD C, BoATright-HoRowitz SL, BoAtright-HorowITZ SS, SIMMONS AM. Discrimination of phase spectra in complex sounds by the bullfrog, Rana catesbeiana. J. Comp. Physiol. A 178:75-87, 1996.

Hall JC, Feng AS. Evidence for parallel processing in the frog's auditory thalamus. J. Comp. Neurol. 258:407-419, 1987.

Hanker JS, Yates PE, Metz CB. A Rustioni A new specific sensitive and non-carcinogenic reagent for demonstration of horseradish peroxidase. Histochem. J. 9:789-792, 1977.

KIM DO, SIRIANNI JG, CHANG SO. Responses of DCN-PVCN neurons and auditory nerve fibers in unanesthetized decerebrate cats to $\mathrm{AM}$ and pure tones: analysis with autocorrelation/power spectrum. Hear Res. 45:95-113, 1990.

LANGNER G. Evidence for neuronal periodicity detection in the auditory system of the guinea fowl: implications for pitch analysis in the time domain. Exp. Brain Res. 52:333-355, 1983.

LANGNER G. Periodicity coding in the auditory system. Hear Res. 60:115-142, 1992.

LANGNER G, SchreINER CE. Periodicity coding in the inferior colliculus of the cat. I. Neuronal mechanisms. J. Neurophysiol. 60:17991822, 1988.

LICKLIDER JCR. A duplex theory of pitch perception. Experientia. 7:128-134, 1951.

LuksCH H, WALKOWIAK W. Morphology and axonal projection patterns of auditory neurons in the midbrain of the painted frog, Discoglossus pictus. Hear Res. 122:1-17, 1998. DOI: 10.1016/S03785955(98) 00081-1.

Lyon R, SHAmma S. Auditory representation of timbre and pitch. Hawkins HL, McMullen TA, Popper AN, Fay RR. Auditory Computation. Springer-Verlag, New York, 1996, 221-270.

MEDDis R, HewITT MJ. Virtual pitch and phase sensitivity of a computer model of the auditory periphery. I. Pitch identification. J. Acoust. Soc. Am. 89:2866-2882, 1991.

MOLLER AR. The use of correlation analysis in processing neuroelec- 
tric data. SCHADE JP, SMITH J. Progress in Brain Research, vol. 33 , Elsevier, New York, 1970, 87-99.

Plomp R. Aspects of Tone Sensation. Academic Press, New York, 1976.

ReEs A, Moller AR. Stimulus properties influencing the responses in inferior colliculus neurons to amplitude-modulated sounds. Hear Res. 27:129-143, 1987.

Rees A, Palmer AR. Neuronal responses to amplitude-modulated and pure-tone stimuli in the guinea pig inferior colliculus and their modification by broadband noise. J. Acoust. Soc. Am. 85:1978-1994, 1989.

RHODE WS. Interspike intervals as a correlate of periodicity pitch in cat cochlear nucleus. J. Acoust. Soc. Am. 97:2414-2429, 1995.

RHODE WS, GREENBERG S. Encoding of amplitude modulation in the cochlear nucleus of the cat. J. Neurophysiol. 71:1797-1825, 1994.

Rose GJ, CAPRANICA RR. Processing amplitude-modulated sounds by the auditory midbrain of two species of toads: matched temporal filters. J. Comp. Physiol. A 154:211-219, 1984.

Rose GJ, CAPRANICA RR. Sensitivity to amplitude modulated sounds in the anuran auditory nervous system. J. Neurophysiol. 53:446$465,1985$.

SCHREINER CE, LANGNER G. Periodicity coding in the inferior colliculus of the cat. II. Topographical organization. J. Neurophysiol. 60:1823-1840, 1988.
SCHWARTZ JJ, SimMONS AM. Encoding of a spectrally-complex communication sound in the bullfrog's auditory nerve. J. Comp. Physiol. A 166:489-500, 1990 .

Simmons AM, Ferragamo M. Periodicity extraction in the anuran auditory periphery, I: 'Pitch-shift' effects. J. Comp. Physiol. A 172:57-69, 1993.

Simmons AM, SchWARTZJJ, FERRAGAMo M. Auditory-nerve representation of a complex communication sound in background noise. J. Acoust. Soc. Am. 91:2831-28441a, 1992.

Simmons AM, Reese G, Ferragamo M. Periodicity extraction in the anuran auditory periphery, II: Phase and temporal fine-structure. J. Acoust. Soc. Am. 93:3374-3389, 1993.

Simmons AM, Buxbaum RC. Neural codes for 'pitch' processing in a unique vertebrate auditory system. Moss CF, SHETTLEWORTH S. Neuroethological Studies of Cognitive and Perceptual Processes Westview Press, Boulder, CO, 1996, 185-228.

Simmons AM, BEAN ME. (2000) Perception of mistuned harmonics in complex sounds by the bullfrog, Rana catesbeiana. J. Comp. Psych., in press.

WILCZYNSKI W. Brainstem auditory pathways in anuran amphibians. Fritzsch B, Ryan MJ, Wilczynsk W, Hetherington TE, WalkowIAK W. Evolution of the Amphibian Auditory System, Wiley, New York, 1988, 209-231. 